\title{
Catalogue and Properties of $\delta$ Scuti Stars in Binaries
}

\author{
Alexios Liakos ${ }^{1 \star}$ and Panagiotis Niarchos ${ }^{2}$ \\ ${ }^{1}$ Institute for Astronomy, Astrophysics, Space Applications and Remote Sensing, National Observatory of Athens, \\ Metaxa \&s Vas. Pavlou St., GR-15236, Penteli, Athens, Hellas \\ ${ }^{2}$ Department of Astrophysics, Astronomy and Mechanics, Faculty of Physics, National 85 Kapodistrian University of Athens, \\ Univ. Campus, GR-15784, Zografos, Athens, Hellas
}

Accepted 201X December XX. Received 201X December XX; in original form 201X October XX

\begin{abstract}
The catalogue contains 199 confirmed cases of binary systems containing at least one pulsating component of $\delta$ Sct type. The sample is divided into subgroups in order to describe the properties and characteristics of the $\delta$ Sct type stars in binaries according to their Roche geometry. Demographics describing quantitatively our knowledge for these systems as well as the distributions of their pulsating components in the Mass-Radius, Colour-Magnitude and Evolutionary Status-Temperature diagrams are presented and discussed. It is shown that a threshold of $\sim 13$ days of the orbital period regarding the influence of binarity on the pulsations is established. Finally, the correlations between the pulsation periods and the orbital periods, evolutionary status, and companion's gravity influence are updated based on the largest sample to date.
\end{abstract}

Key words: (stars:) binaries (including multiple): close-stars: fundamental parameters-stars: variables: $\delta$ Scuti-(stars:) binaries: eclipsing-stars: fundamental parameters

\section{INTRODUCTION}

The single pulsating stars of $\delta$ Scuti type range between AF spectral types and have mass values between $1.4-3 \mathrm{M}_{\odot}$. They lie inside the classical instability strip and their luminosity classes range between III-V (Breger 2000). They potentially exhibit radial and non radial pulsations with frequencies ranging between 3-80 cycle $\mathrm{d}^{-1}$ (Breger 2000) and can oscillate in both $p$ and $g$ modes. The origin and the evolution of their intrinsic oscillations are in general explained by the $\kappa$-mechanism mostly due to partial ionization of HeII (Houdek et al. 1999; Balona et al. 2015, and references therein) and with a small contribution of the combined $\mathrm{HI}$ and HeI ionization zone (Castor 1968). However, recent results by Antoci et al. (2014) and Grassitelli et al. (2015) show that the $\kappa$-mechanism is insufficient to explain all the frequency modes, especially the high-order, non-radial ones. According to these works the turbulent pressure in the Hydrogen convective zone can be considered as an additional pulsations driving mechanism.

Binary systems are stellar objects that can be considered as the most powerful tools for astronomers to derive absolute stellar parameters (e.g. masses, radii, luminosities) and to create stellar evolutionary models. The eclipsing systems, in particular, provide the means for accurate determi-

^ E-mail: alliakos@noa.gr nation of those parameters, which can be easily calculated by modelling their light and radial velocity curves (hereafter RVs). Moreover, binary systems also provide an extra tool, namely the orbital period analysis (i.e. $O-C$ or $E T V$ analysis), which is essential for understanding the binary interaction and the physical mechanisms (i.e. mass transfer) that modulate their orbital period.

$\delta$ Scuti stars in binary systems show slight difference in comparison with the single ones regarding the evolutionary stage, since they are located mostly inside the Main Sequence band (Liakos et al. 2012) and only few of them have left it. On the other hand, single and binary $\delta$ Scuti stars show similar oscillation properties. The first notification of this kind of systems was made by Mkrtichian et al. (2002), who introduced the Algol-type mass accreting pulsators of (B)A-F type as a new class of stellar systems that is commonly referred as ' $O E A$ stars' (i.e. oscillating eclipsing systems of Algol type). It should be noticed that this definition is general and can also be applied to binaries that include pulsating stars of another type (e.g. $\gamma$ Doradus, $\beta$ Cephei, RR Lyrae, Classical Cepheids), but until 2002 systematic works had been made only for binaries with $\delta$ Scuti stars. According to Mkrtichian et al. (2003) and Soydugan et al. (2006b) the discrepancy in evolution between single and binary members $\delta$ Scuti stars can be explained by the mass transfer and the tidal distortions caused by the companion of the pulsator during or even before its Main Sequence life. 
The history of the pulsating stars in binaries covers the last $\sim 25$ years, while, in particular for the binary $\delta$ Scuti stars, during the last $\sim 10$ years a lot of research papers have been published showing significant evolution in our understanding about their properties. In general, the systematic cataloguing of binary pulsators began from Szatmary (1990), who published a catalogue with all the known pulsating stars that potentially belong to binary systems. Ten years later, due to the increasing number of discoveries, a need for further classification according to the pulsations type was revealed. Rodríguez et al. (2000) published a catalogue with all the known $\delta$ Sct stars and pointed out that 86 of them belong to binary or multiple systems. Rodríguez \& Breger (2001), based on the catalogue of Rodríguez et al. (2000), listed tables with $15 \delta$ Sct stars in total that are members of binaries. The first results concerning physical properties of the binary $\delta$ Sct stars were published by Soydugan et al. (2006b), who noticed for the first time the connection between pulsation and orbital periods for eclipsing binaries with a $\delta$ Sct member using a sample of 20 systems. A few months later, Soydugan et al. (2006c) published lists with confirmed and candidate such systems. A few years later, Zhou (2010) published a catalogue containing 89 systems with an oscillating member and distinguished them according to their pulsational properties. Liakos et al. (2012), after a long term observational campaign on more than 100 eclipsing systems-candidates for including a $\delta$ Sct component, published an updated catalogue with 75 confirmed cases and derived new correlations between their fundamental stellar characteristics. Zhang et al. (2013) presented the first theoretical approach for the connection between orbital and dominant pulsation periods for specifically $\delta$ Sct stars in binaries. Chang et al. (2013) published the most recent catalogue of $\delta$ Sct stars (1578 in total) including 141 cases that are members of binary or multiple systems. Finally, Liakos \& Niarchos $(2015,2016)$ published an updated catalogue containing the currently known binary $\delta$ Sct stars and noticed for first time that there is a threshold in the orbital period beyond which there is no influence of the binarity to the pulsations.

Similar studies for other pulsating stars in binaries were made by Cakırlı \& İbanoğlu (2016), who found similar correlations between pulsation-orbital periods and pulsation period - $\log g$ for the $\gamma$ Dor-binary members, and by Skarka et al. (2016) who published a review for RR Lyrae type pulsators in binary systems.

The present catalogue contains all the currently known binaries with at least one $\delta$ Sct member. Each system included here was extensively checked by using all the available information from the literature, was characterized and categorized according to its Roche geometry. The novelty of this catalogue in comparison with the respective previous ones (e.g. Soydugan et al. 2006c; Liakos et al. 2012; Liakos \& Niarchos 2015, 2016) is that it also contains systems on wide orbits (i.e. visual binaries) as well as systems with unseen companions (i.e. their binarity was detected through dominant pulsation frequency modulations, see Shibahashi \& Kurtz 2012). Each individual case is given the corresponding literature references that contain exactly the information for its properties (e.g. basic orbital and physical parameters, pulsation analysis). This provides the means for the reader to directly check each value listed as well as, in some cases, the chronicle of the system. In addition, the apparent limit of $\sim 13$ days (Liakos \& Niarchos 2015,2016 ), that separates the systems whose intrinsic pulsations of their $\delta$ Sct stars are affected by the binarity, seems to be well established. Given that the present sample is much larger than all those used in previous studies for the correlations between fundamental stellar characteristics of these systems, updated ones are also presented. Finally, one of the most important contributions of this paper is the list with the ambiguous cases, which can be used for future target selection for observations with the aim to check them for pulsations and/or binarity.

\section{THE CATALOGUE}

The catalogue consists of five lists given in Tables 1-5 of this paper. It contains all of the currently known binaries with a $\delta$ Sct component, ambiguous ones, and others that were incorrectly reported as being of this type. This catalogue is online $^{1}$ and is frequently updated.

Tables 1, 2, and 3 include all of the currently known binary systems (199 systems) hosting at least one pulsating component of $\delta$ Sct type (203 pulsators). The whole sample is divided into two main groups according to the orbital period $\left(P_{\text {orb }}\right)$ value of the systems. Particularly, Table 1 includes the 118 systems with $P_{\text {orb }}<13$ days, while Table 2 those with $P_{\text {orb }}>13$ days. This separation of the sample is based on the arguments given by Liakos \& Niarchos $(2015,2016$, i.e. the pulsations are not affected by the binarity in systems with $P_{\text {orb }}>13 \mathrm{~d}$ ) and is further discussed in Section 3. The sample of each table is further subdivided according to the Roche geometry of the systems, since the properties of each subgroup are different (see Sections 4, 5). In particular, they are divided into 'Semidetached' (the non-pulsating star fills its Roche lobe), 'Detached', and 'Unclassified' systems (i.e. unknown geometrical configuration). Tables 1 and 2 include: the name of the system, its orbital period $\left(P_{\text {orb }}\right)$, the dominant pulsation frequency $\left(f_{\text {dom }}\right)$ (i.e. the frequency with the largest amplitude) of its $\delta$ Sct star and its corresponding amplitude $(A)$ in a given filter (e.g. $B, V, b, y, W=$ white light, $K p=$ Kepler, $H p=$ HIPPARCOS, $C o R=\mathrm{CoRoT}$ ), and the system's type of variation $(T o V)$ (i.e. $E=$ Eclipsing Binary, $S B=$ Spectroscopic Binary; $1=$ Single line; $2=$ Double line, $E V=$ Ellipsoidal Variable, $V i s=$ Visual Binary, $O-$ $C=$ system with its $\delta$ Sct star showing cyclic variation of its dominant pulsation period). Moreover, the absolute parameters of the $\delta$ Sct $\operatorname{star}\left(M_{\text {pul }}, R_{\text {pul }}, T_{\text {pul }}, \log g_{\text {pul }}\right)$ as well as those of its companion $\left(M_{\text {comp }}, R_{\text {comp }}\right)$ are also listed. The symbols $M, R, T, \log g$ correspond to the standard quantities i.e. mass, radius, temperature and gravity acceleration value, respectively. Moreover, the orbital separation of the system's components $(a)$ and their mass ratio $(q)$ are also presented. In many cases some of the values of the absolute parameters are marked with an asterisk $\left(^{*}\right)$. In general, many authors, in absence of RVs measurements, assume the mass value of the primary component according to its spectral type and its luminosity class (i.e. MS

\footnotetext{
1 http://alexiosliakos.weebly.com/catalogue.html
} 
star). The rest parameters, however, follow this assumption (e.g. Soydugan et al. 2009, 2011; Liakos \& Niarchos 2013b; Lee et al. 2016a,b; Guo et al. 2016). Table 3 contains binaries with a $\delta$ Sct component for which no exact $P_{\text {orb }}$ values have been determined so far. These systems are also considered as unclassified ones, but they are not used in any of the following correlations (Section 4). In the last column of Tables 1-3 the respective reference codes for each system are given and they are fully explained at the bottom of the tables. For many systems additional comments (e.g. cross-identification names, special properties) are also given as tablenotes and they are very useful for literature search. The orbital period values for the majority of the eclipsing binaries were taken from Kreiner et al. (2001); Kreiner (2004), while for the Kepler systems from Slawson et al. (2011) and Kirk et al. (2016). In Tables 1-3 the errors are given alongside values in parentheses and they correspond to the last $\operatorname{digit}(\mathrm{s})$.

Table 4 includes the cases which had been previously reported as binaries with a $\delta$ Sct member, but now they are considered as ambiguous ones. The challenging reasons for these cases to be members of the binary $\delta$ Sct stars group are: a) questionable binarity, b) questionable pulsational behaviour, and c) incorrect classification in the first place. The majority of these cases were reported in the catalogues of Szatmary (1990), Rodríguez et al. (2000), Zhou (2010), and Chang et al. (2013), while the most of the Kepler stars/systems were listed by Uytterhoeven et al. (2011) and Bradley et al. (2015). Table 5 contains the rejected cases of binaries with a $\delta$ Sct component. Most of them were also listed in the aforementioned catalogues, but it was found that either they are not binaries or they do not exhibit pulsations of $\delta$ Sct type. Each individual case of these tables was examined carefully and the final comments are based on an extensive literature search. The first column contains the name of the star/system, the second one comments for each case, while in the last one are given the relevant reference codes.

In all tables the objects are presented in alphabetical order according to either their GCVS (General Catalogue of Variable Stars) designation or to their individual catalogue name. In general, the reference codes are written in the form ' $X X X N N$ ', where ' $X X X$ ' corresponds to the first three letters of the first author's surname and ' $N N$ ' to the year of the publication. The reference codes in the last columns of the tables are given in chronological order. At the bottom of the tables, the references are given in alphabetical order. It should be mentioned that only the most important references are given for each case and it is recommended for the reader to also check the references therein for a more comprehensive view.

The results from light curve modelling and pulsation analysis for KIC 06629588 and KIC 06669809 were briefly presented by Liakos \& Niarchos (2016), while the results (Roche geometry model and pulsation analysis) for KIC 10619109, KIC 11175495, KIC 10686876, and WX Dra $(=\mathrm{KIC} 10581918)$ are presented here for first time. For all these systems a detailed analysis will be presented in a future paper. The detailed results for GQ Dra are not yet published, but the value of $f_{\text {dom }}$ was kindly provided through a private communication with B. Ulas. Gaulme \& Guzik
(2014) published preliminary results of the frequency analysis for KIC 04570326, KIC 05872506, KIC 06541245, KIC 11401845, and KIC 11973705 without giving the exact values of $f_{\text {dom }}$, but only their frequency range. The systems which have been found to include two pulsators of $\delta$ Sct type (RS Cha, $\delta$ Del, KIC 09851944, and KIC 10080943) are given two tablelines, one for each pulsating component (referred as A and B). It should to be noted that the light curves of all Kepler binaries mentioned in this study are available online ${ }^{2}$.

\section{DEMOGRAPHICS}

In this section we present various demographical diagrams showing distributions of the binaries with a $\delta$ Sct component according to their physical and/or geometrical properties (e.g. Mass, radius, Roche geometry).

In Fig. 1 the demographics of the sample of the binary $\delta$ Sct stars according to the Roche geometry configuration of the system in which they belong is presented. In the same plot, similar demographics are also given for the binary $\delta$ Sct stars in systems with $P_{\text {orb }}<13$ days, since they are the only ones that are used for the correlations of physical parameters in the next section. In Fig. 2 the demographics of the binary $\delta$ Sct stars in systems with $P_{\text {orb }}<13$ days and with known absolute parameters is also presented. In this graph, the sample is divided into three different groups according to the information used for the calculation of their absolute parameters. In particular, the first group (SB2+E) contains the $\delta$ Sct stars in systems which are both eclipsing and double-line spectroscopic binaries and whose their absolute parameters were calculated from data derived from the analysis of RVs and light curves. The second group (E or $\mathrm{EV}$ ) includes the $\delta$ Sct stars in eclipsing and ellipsoidal systems for which there is no spectroscopic information so far. The calculation of their absolute parameters is based only on the light curves solution and on assumptions for the mass of the primary star according to theoretical models. The third group (SB1+E or SB1 $(2)+\mathrm{EV})$ hosts the $\delta$ Sct stars in systems which are either eclipsing and single-line spectroscopic binaries or ellipsoidal and single or double-line spectroscopic binaries. For these systems, an assumption for one absolute parameter (e.g. the mass of the primary star) has been made with the others following it.

The unclassified systems, regardless of their $P_{\text {orb }}$ value, are cases with unknown Roche geometry. Preventing reasons for their classification are: a) their light curves have not been obtained yet, b) their light curves are incomplete, c) their light curves are available but no analysis has been made, and d) the system is not eclipsing.

Systems with relatively long $P_{\text {orb }}$ values (e.g. $>$ 100 days) can also be potentially considered as detached ones, but for reasons of homogeneity of the sample (i.e. lack of evidence) they are simply listed as unclassified. On the other hand, systems in eccentric and relatively wide (i.e. $P_{\text {orb }}$ ranging from tenths to millions of days) orbits (marked with the superscript ' $e$ ' in Tables 1-2) are considered directly as

\footnotetext{
2 http://keplerebs.villanova.edu/
} 


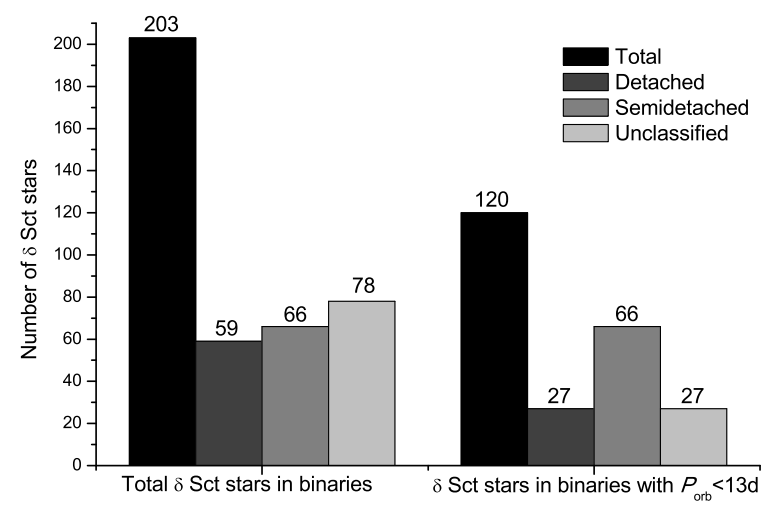

Figure 1. Demographics of $\delta$ Sct stars in binaries according to the Roche geometry (detached, semi-detached, unclassified) of the systems. Left columns refer to $\delta$ Sct stars of all systems, while the right ones to those that belong to systems with $P_{\text {orb }}<13$ days.

detached ones (e.g. BS Aqr, IK Peg). The latter assumption is based on the works of Petrova \& Orlov (1999) and Davis et al. (2013), who concluded that eccentric semidetached systems are rather rare and their majority have relatively short orbital periods (i.e. they range between 0.5-12 days).

According to our literature search, 56 cases are doubtful for being binaries with a $\delta$ Sct member. In particular, for 5 cases the binarity has not yet been proven, for 26 systems the pulsations occurrence is doubtful, 20 cases are indeed binaries that host a pulsator but the type of pulsations needs further investigation, and 5 cases are ambiguous concerning both the binarity and the existence of pulsations. The demographics of all these cases are shown in Fig. 3, while details for each individual are listed in Table 4.

On the other hand, another 42 cases are rejected from being members of this group (Table 5), either because they were proven to be single stars or they are binaries but: a) none of their components exhibit pulsations, b) their pulsations type is different than that of $\delta$ Sct stars, and c) they have properties other than pulsational (e.g. flare stars).

Fig. 4 shows the $P_{\text {orb }}-P_{\text {puls }}$ diagram for all of the currently known $\delta$ Sct stars in binaries. In this plot 147 out of 203 cases are included with their respective values to be given in Tables 1 and 2. 56 binary $\delta$ Sct stars are omitted because either their $P_{\mathrm{pul}}$ or the $P_{\text {orb }}$ of their systems have not been determined so far (see Section 2 for details). The most important note that can be made for this diagram is the obvious existence of two subgroups. The first one includes the cases with the smaller values of $P_{\text {orb}}$, while the second one those with larger $P_{\text {orb }}$. The limit that has been set at $P_{\text {orb }} \sim 13$ days is arbitrary, it may be apparent and its exact value needs further clarification. However, the strong linear correlation between $P_{\text {orb }}-P_{\text {pul }}$ for the systems with $P_{\text {orb }}<13$ days is obvious even by naked eye, while for those with larger $P_{\text {orb }}$ values these quantities seem to be uncorrelated. More systems with $10<P_{\text {orb }}<30$ days are needed for a better estimation of this boundary. With more data points in this area, maybe this limit can reach the value of $P_{\text {orb }}=30-40$ days (i.e. $\log P_{\text {orb }} \sim 1.5-1.6$ ). Therefore, for the correlations presented in the next section, only the properties of systems with $P_{\text {orb }}<13$ days are taken into account.

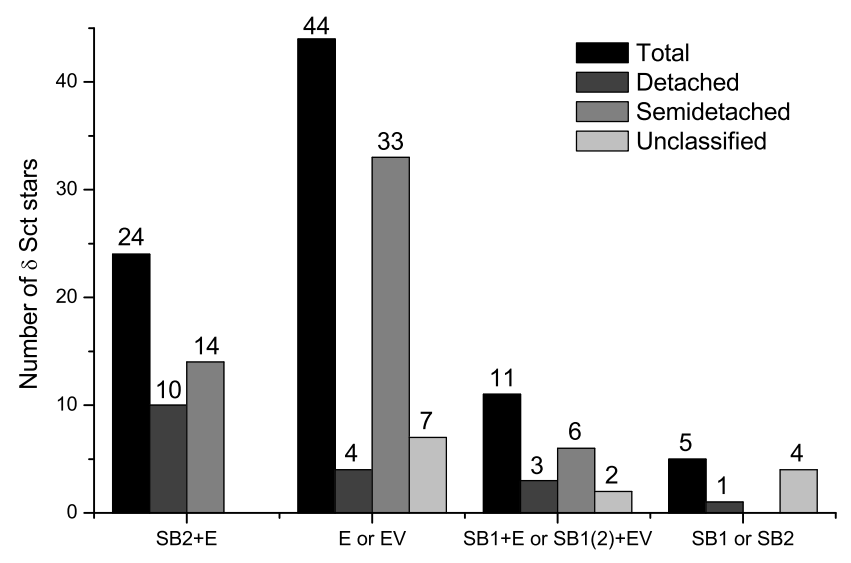

Figure 2. Demographics of $\delta$ Sct stars in binaries (with $P_{\text {orb }}<$ 13 days) and with known absolute parameters distributed according to the geometrical type of the systems. The sample is further subdivided according to the available information for the calculation of the absolute parameters. For details see Section 3.

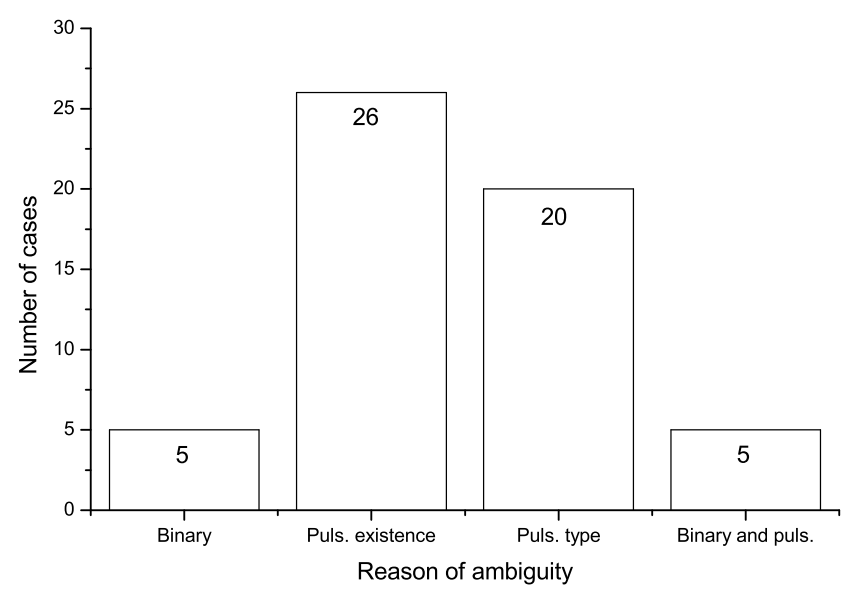

Figure 3. The distribution of doubtful cases of binaries with a $\delta$ Sct component according to the reason of ambiguity. For details see Table 4 .

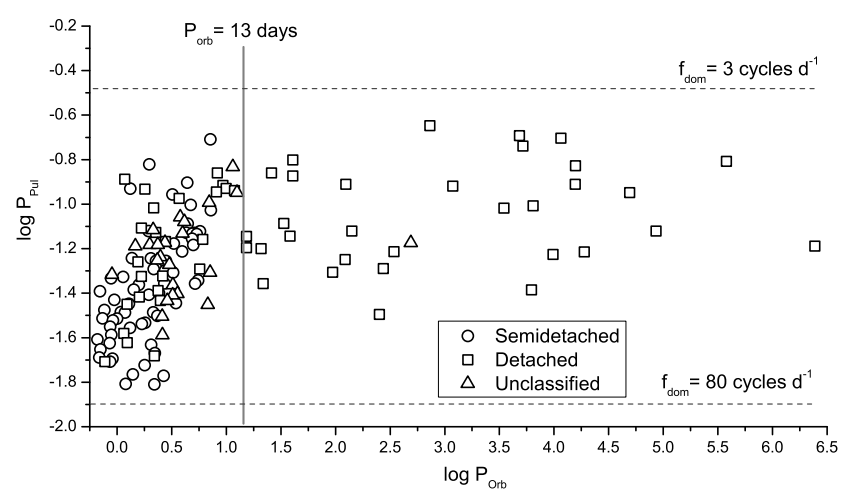

Figure 4. The $P_{\text {orb }}-P_{\text {pul }}$ diagram of binary $\delta$ Sct stars. The limit of $P_{\text {orb }}=13$ days is set for the separation of the two subgroups. Grey dash lines show the limits of the frequency range of $\delta$ Sct stars (3-80 cycle $\left.\mathrm{d}^{-1}\right)$. 
Table 1. List of binaries with $P_{\text {orb }}<13$ days containing a $\delta$ Sct component.

\begin{tabular}{|c|c|c|c|c|c|c|c|c|c|c|c|c|c|}
\hline Name & $\begin{array}{c}P_{\text {orb }} \\
\text { (days) }\end{array}$ & $\begin{array}{c}f_{\text {dom }} \\
{\text { (cycle } \mathrm{d}^{-1} \text { ) }}^{\text {con }}\end{array}$ & $\begin{array}{c}A \\
(\mathrm{mmag})\end{array}$ & $\mathrm{ToV}$ & $\begin{array}{c}M_{\text {pul }} \\
\left(\mathrm{M}_{\odot}\right)\end{array}$ & $\begin{array}{l}R_{\text {pul }} \\
\left(\mathrm{R}_{\odot}\right)\end{array}$ & $\begin{array}{c}T_{\text {pul }} \\
(\mathrm{K})\end{array}$ & $\begin{array}{l}\log g_{\mathrm{pul}} \\
\left(\mathrm{cm} \mathrm{s}^{-2}\right)\end{array}$ & $\begin{array}{c}M_{\text {comp }} \\
\left(\mathrm{M}_{\odot}\right)\end{array}$ & $\begin{array}{c}R_{\text {comp }} \\
\left(\mathrm{R}_{\odot}\right)\end{array}$ & $\begin{array}{c}a \\
\left(\mathrm{R}_{\odot}\right)\end{array}$ & $q$ & References \\
\hline \multicolumn{14}{|c|}{ Semi-detached systems } \\
\hline J050634.16-353648.4 $4^{\text {cat } 1}$ & $5.104238(5)$ & 13.45 & $65(7)(V)$ & $\mathrm{SB} 2+\mathrm{E}$ & $1.73(11)$ & $2.40(7)$ & $7000 *(200)$ & $3.91(2)$ & $0.41(3)$ & $4.2(1)$ & $16.1(3)$ & $0.236(4)$ & NOR16 \\
\hline CZ Aqr & $0.862752(3)$ & $35.508(2)$ & $3.7(5)(B)$ & $\mathrm{E}$ & $2.0^{*}$ & $1.9(1)$ & $8200^{*}$ & $4.2(1)$ & $0.98(1)$ & $1.8(1)$ & $5.62(1)$ & $0.49(1)$ & LIA12 \\
\hline DY Aqr & $2.159679(1)$ & $19.5645(4)$ & $14.1(7)(V)$ & $\mathrm{SB} 1+\mathrm{E}$ & $1.8(2)$ & $2.1(1)$ & $7625(125)$ & $4.1(1)$ & $0.55(4)$ & $2.7(1)$ & $9.4(5)$ & $0.31(2)$ & SOY09, ALF14 \\
\hline RY Aqr & $1.966565(1)$ & $6.62(8)$ & $32.7(4)(B)$ & $\mathrm{SB} 2+\mathrm{E}$ & $1.27(7)$ & $1.39(7)$ & $7650^{*}$ & $4.3(6)$ & $0.26(2)$ & $1.9(1)$ & $7.6(1)$ & $0.204(7)$ & POP89, MAN16 \\
\hline QY Aql & $7.229560(1)$ & $10.6561(1)$ & $11.8(2)(B)$ & $\mathrm{E}$ & $1.6^{*}(2)$ & $4.1(2)$ & $7300^{*}$ & $3.4(1)$ & $0.4(1)$ & $5.4(2)$ & $20.3(5)$ & $0.25(2)$ & LIA12, LIA13b \\
\hline V729 Aql & $1.281905(1)$ & $28.034(1)$ & $4.2(4)(W)$ & E & $1.5^{*}(2)$ & $1.96(1)$ & $6900^{*}$ & $4.0(1)$ & $0.7(1)$ & $2.03(1)$ & $6.6(1)$ & $0.44(1)$ & LIA14 \\
\hline V1464 Aql & 0.69777 & $24.621(1)$ & $17.7(3)(B)$ & $\mathrm{SB} 1+\mathrm{EV}$ & $2.2(4)$ & $2.5(6)$ & $7100^{*}$ & $4.0(2)$ & $0.3(1)$ & $1.0(2)$ & $4.6(1)$ & $0.15(1)$ & POU04, DAL13, LIA13a \\
\hline XZ Aql & $2.1392073(7)$ & $30.631(1)$ & $5.4(4)(B)$ & $\mathrm{SB} 2+\mathrm{E}$ & $2.42(14)$ & $2.45(7)$ & 8770 & $4.04(6)$ & $0.45(6)$ & $2.43(6)$ & $9.9(2)$ & $0.184(4)$ & SOY16, ZOL16 \\
\hline EW Boo & $0.9063521(3)$ & $49.4496(1)$ & $21.3(5)(V)$ & $\mathrm{SB} 2+\mathrm{E}$ & $2.00(4)$ & $1.88(2)$ & $8970^{*}$ & $4.19(1)$ & $0.327(8)$ & $1.23(1)$ & $5.22(4)$ & $0.164(1)$ & SOY08, SOY10, ZHA15a, DOG15 \\
\hline YY Boo & $3.93305(1)$ & $16.31828(2)$ & $58.4(2)(B)$ & $\mathrm{E}$ & & & $8000^{*}$ & & & & $0.22(1)$ & $0.29(1)$ & HAM10 \\
\hline Y Cam & $3.305766(3)$ & $15.0469(1)$ & $8.2(2)(V)$ & $\mathrm{SB} 2+\mathrm{E}$ & $2.08(9)$ & $3.14(5)$ & $8000(250)$ & $3.76(2)$ & $0.48(3)$ & $3.33(5)$ & $13.1(2)$ & $0.23(2)$ & KIM03, SOY06b, ROD10, HON15 \\
\hline $\mathrm{R} \mathrm{CMa}$ & $1.1359536(7)$ & 21.231 & $8.8(B)$ & $\mathrm{SB} 2+\mathrm{E}$ & $1.67(8)$ & $1.78(3)$ & $7300^{*}$ & $4.16(2)$ & $0.22(7)$ & $1.22(7)$ & $5.8(3)$ & $0.13(4)$ & MKR00, SOY06b, BUD11 \\
\hline TY Cap & $1.423458(2)$ & $24.222(1)$ & $18.5(7)(B)$ & $\mathrm{E}$ & $2.0^{*}$ & $2.5(1)$ & $8200^{*}$ & $3.9(1)$ & $1.05(1)$ & $2.5(1)$ & $7.09(3)$ & $0.52(1)$ & LIA09, LIA12 \\
\hline $\mathrm{AB}$ Cas & $1.3668918(2)$ & 17.492 & $26.6(B)$ & $\mathrm{E}$ & $1.87(17)$ & $1.90(6)$ & $8000^{*}$ & $4.16(4)$ & $0.38(3)$ & $1.63(5)$ & $7.0(1)$ & $0.20(2)$ & RO04, SOY06b, ABE07, KHA12 \\
\hline IV Cas & $0.9985078(2)$ & $32.692(1)$ & $3.4(2)(B)$ & $\mathrm{E}$ & $1.98(10)$ & $2.13(4)$ & 8500 & $4.08(3)$ & $0.81(4)$ & $1.80(3)$ & $6.06(2)$ & $0.408(1)$ & KIM05d, KIM10 \\
\hline RZ Cas & $1.195241(8)$ & $64.1965(7)$ & $2.7(3)(B)$ & $\mathrm{SB} 2+\mathrm{E}$ & $2.01(2)$ & $1.61(1)$ & $8907(15)$ & $4.328(4)$ & $0.69(1)$ & $1.93(3)$ & $6.59(3)$ & $0.342(2)$ & OHS01, SOY06a, SOY07, GOL07, TKA09 \\
\hline V1264 $\mathrm{Cen}^{a 1}$ & $5.3504751(6)$ & $13.62(2)$ & $350.0(\mathrm{~V})$ & $\mathrm{SB} 2+\mathrm{E}$ & $1.49(2)$ & $2.35(2)$ & 7500 & $3.87(1)$ & $0.33(2)$ & $4.04(1)$ & $16.1(3)$ & $0.22(2)$ & CHR07 \\
\hline XX Cep & $2.33736(7)$ & 31.77 & $2.9(2)(B)$ & $\mathrm{SB} 2+\mathrm{E}$ & $2.49(6)$ & $2.27(2)$ & 7946 & $4.12(1)$ & $0.38(1)$ & $2.43(2)$ & $10.5(1)$ & $0.151(2)$ & ANG06, LEE07, HOS14, KOO16 \\
\hline WY Cet & $1.939689(1)$ & $13.211(1)$ & $7.7(3)(B)$ & $\mathrm{E}$ & $1.7^{*}$ & $2.2(1)$ & $7500(150)$ & $4.0(1)$ & $0.44(1)$ & $2.3(1)$ & $8.7(3)$ & $0.26(1)$ & LIA09, LIA12 \\
\hline UW Cyg & $3.450759(1)$ & $27.841(2)$ & $1.9(2)(B)$ & $\mathrm{E}$ & $1.9^{*}$ & $2.2(1)$ & $8000(150)$ & $4.0(1)$ & $0.26(1)$ & $2.9(1)$ & $12.7(2)$ & $0.14(1)$ & LIA12 \\
\hline V346 Cyg & 2.7433338 & 19.912 & $30.0(B)$ & $\mathrm{E}$ & 2.3 & 3.8 & 8353 & 3.6 & 1.83 & 4.74 & 13.55 & 0.8 & BUD04, KIM05b, SOY06b, HAN14 \\
\hline V469 Cyg & $1.3124995(8)$ & 35.971 & $20.0(V)$ & $\mathrm{E}$ & & & & & & & & & CAT04 \\
\hline BW Del & $2.423133(6)$ & $25.100(1)$ & $29(2)(B)$ & $\mathrm{E}$ & $1.5^{*}(2)$ & $2.1(1)$ & $7000^{*}$ & $4.0(1)$ & $0.3(1)$ & $2.2(1)$ & $9.3(4)$ & $0.16(2)$ & LIA13b \\
\hline GQ Dra & $0.7659023(4)$ & $29.860(1)$ & $2.2(5)(B)$ & $\mathrm{E}$ & & & & & & & & & private commun. \\
\hline HL Dra & $0.9442760(2)$ & $26.914(1)$ & $3.0(2)(B)$ & $\mathrm{E}$ & $2.5(2)$ & $2.5(4)$ & 8200 & $4.0(1)$ & $0.9(1)$ & $1.8(3)$ & $6.1(2)$ & $0.37(1)$ & LIA12 \\
\hline SX Dra & $5.169412(1)$ & $22.7423(1)$ & $17.3(4)(B)$ & $\mathrm{E}$ & $1.75^{*}$ & $2.42(7)$ & $7762^{*}$ & $3.91(4)$ & $0.75(5)$ & $5.36(5)$ & $17.5(1)$ & $0.430(1)$ & DIM10, SOY13 \\
\hline TW Dra & $2.806791(3)$ & 17.986 & $10.0(B)$ & $\mathrm{SB} 2+\mathrm{E}$ & $2.2(1)$ & $2.64(4)$ & $7815(92)$ & $3.93(3)$ & $0.93(5)$ & $3.66(6)$ & $12.48(2)$ & $0.430(2)$ & KIM03, ZEJ10, TKA10, BOZ13 \\
\hline TZ Dra & $0.866033(1)$ & $50.993(2)$ & $3.7(2)(B)$ & $\mathrm{E}$ & $1.8^{*}(2)$ & $1.7(1)$ & $7800^{*}$ & $4.2(1)$ & $0.6(1)$ & $1.5(1)$ & $5.2(2)$ & $0.31(3)$ & SOY06b, LIA13b \\
\hline WX Dra ${ }^{a 2}$ & $1.801863(3)$ & $34.0782(1)$ & $1.171^{k}(1)(K p)$ & $\mathrm{E}$ & & & $7800^{*}$ & $3.918^{*}$ & & & & & PET12, this paper \\
\hline AS Eri & $2.664145(3)$ & $59.03116(5)$ & $\begin{array}{l}3.4(4)(V) \\
3\end{array}$ & $\mathrm{E}$ & $1.93^{*}$ & $1.57^{*}$ & $8470^{*}$ & $\begin{array}{c}0.710 \\
4.3\end{array}$ & & & & & MKR04, NAR13 \\
\hline TZ Eri & $2.606113(3)$ & $18.7174(3)$ & $8.3(1)(B)$ & $\mathrm{SB} 2+\mathrm{E}$ & $1.97(6)$ & $1.69(3)$ & $9307(20)$ & $4.28(3)$ & $0.37(1)$ & $2.60(4)$ & $10.6(2)$ & $0.177(1)$ & BAR98, LIA08 \\
\hline GSC 3889-0202 & $2.71066(8)$ & 22.676 & $50.0(V)$ & $\mathrm{SB} 1+\mathrm{E}$ & & & 7750 & 3.9 & & & & & DIM08b \\
\hline GSC $4293-0432^{a 3}$ & $4.3844(2)$ & 8.0 & $40.0(B)$ & $\mathrm{SB} 1+\mathrm{E}$ & & & 7750 & & & & & & DIM09b \\
\hline GSC 4588-0883 & $3.25855(9)$ & 20.284 & $15.0(R)$ & $\mathrm{SB} 1+\mathrm{E}$ & & & 7650 & 3.9 & & & & 0.16 & DIM09a \\
\hline $\begin{array}{l}\text { BO Her } \\
\text { BO-1000 }\end{array}$ & $4.272834(2)$ & $13.430(1)$ & $68.0(3)(B)$ & $\mathrm{E}$ & $1.8^{*}(2)$ & $2.5(1)$ & $7800^{*}$ & $3.9(1)$ & $0.4(1)$ & $3.8(1)$ & $14.8(1)$ & $0.22(2)$ & SUM07, LIA13b \\
\hline CT Her & $1.7863793(5)$ & $52.9366(1)$ & $3.3(1)(B)$ & $\mathrm{SB} 1+\mathrm{E}$ & $2.28(1)$ & $2.06(6)$ & $8200^{*}$ & $4.17(2)$ & $0.29(4)$ & $1.87(8)$ & $8.69(3)$ & $0.1267(6)$ & KIM04b, LAM11 \\
\hline EF Her & $4.729170(3)$ & 10.07 & $69.0(B)$ & $\mathrm{E}$ & $1.82^{*}$ & 2.8 & $7300^{*}$ & 3.8 & 0.27 & & 15.14 & 0.15 & KIM04b, SEN08, SOY13 \\
\hline LT Her & $1.0840335(4)$ & $30.521(3)$ & $6.0(B)$ & $\mathrm{E}$ & & & $9400^{*}$ & & & & & & LIA13b, STR16 \\
\hline TU Her & $2.266944(2)$ & $18.0(2)$ & $5.0(V)$ & $\mathrm{E}$ & & & & & & & & & LAM04 \\
\hline RX Hya & $2.2816596(9)$ & 19.38 & $7.0(B)$ & $\mathrm{SB} 1+\mathrm{E}$ & 2.72 & 2.17 & 8770 & 4.2 & 0.66 & 2.93 & 11.2 & 0.243 & LUC71, VYA89, KIM02, KIM03 \\
\hline KIC 06220497 & $1.32317(3)$ & $8.5175(3)$ & $4.6(2)(K p)$ & E & $1.60^{*}(8)$ & $2.69(6)$ & $7270 *(6)$ & $3.78(3)$ & $0.39(2)$ & $1.68(4)$ & $6.53(2)$ & $0.242(3)$ & GAU14, LEE16a \\
\hline KIC 06669809 & $0.7337378(7)$ & $32.5636(2)$ & $0.560^{k}(1)(K p)$ & $\mathrm{E}$ & $1.8^{*}(3)$ & $2.5^{*}(9)$ & $7462 *(261)$ & $3.9 *(4)$ & & & & & HUB14, LIA16 \\
\hline KIC 10619109 & $2.045183(3)$ & $42.801(1)$ & $0.606^{k}(4)(K p)$ & $\mathrm{E}$ & $1.5 *(3)$ & $2.1^{*}(8)$ & $7138 *(284)$ & $3.9 *(4)$ & & & & & HUB14, this paper \\
\hline KIC 11175495 & $2.191027(3)$ & $64.4434(1)$ & $3.016^{k}(5)(K p)$ & $\mathrm{E}$ & $2.0^{*}(3)$ & $3.1 *(5)$ & $8293^{*}(290)$ & $3.8^{*}(4)$ & & & & & HUB14, this paper \\
\hline AU Lac & $1.3924354(3)$ & $58.217(1)$ & $5.0(3)(B)$ & $\mathrm{E}$ & $2.0^{*}$ & $1.8(1)$ & $8200^{*}$ & $4.2(1)$ & $0.6(1)$ & $2.1(1)$ & $7.4(2)$ & $0.30(1)$ & $\begin{array}{l}\text { LIA12 } \\
\text { Lins paper }\end{array}$ \\
\hline WY Leo & 4.9858404 & $15.2528(1)$ & $11(1)(V)$ & $\mathrm{E}$ & 2.31 & 3.26 & 9640 & 3.78 & 1.41 & 2.65 & 19.02 & 0.61 & BRA80, DVO09 \\
\hline Y Leo & $1.686086(1)$ & $34.4834(6)$ & $4.1(2)(V)$ & $\mathrm{E}$ & 2.29 & 1.90 & 8700 & 4.24 & 0.74 & & 8.63 & 0.32 & TUR08, TUR11, POP11 \\
\hline RR Lep & $0.9154229(3)$ & $33.2802(4)$ & $9.6(4)(B)$ & $\mathrm{E}$ & $1.8^{*}(2)$ & $2.2(1)$ & $7800^{*}$ & $4.0(1)$ & $0.4(1)$ & $1.4(2)$ & $5.3(2)$ & $0.23(2)$ & DVO09, LIA13b \\
\hline CL Lyn & 1.58606 & $23.051(1)$ & $7.3(3)(B)$ & $\mathrm{E}$ & $2.0^{*}$ & $2.5(1)$ & $8200^{*}$ & $3.9(1)$ & $0.38(3)$ & $1.9(1)$ & $7.9(2)$ & $0.19(2)$ & LIA12 \\
\hline VY Mic & $4.436373(2)$ & $12.23411(3)$ & 19.4(2) (V) & $\mathrm{E}$ & 2.39 & 2.24 & 8500 & 4.12 & 1.96 & 4.43 & 19.01 & 0.82 & BUD04, PIG07 \\
\hline V2365 Oph & $4.86560(1)$ & 14.286 & $50.0(\mathrm{~V})$ & $\mathrm{SB} 2+\mathrm{E}$ & $1.97(2)$ & $2.19(1)$ & $9500(200)$ & $4.051(3)$ & $1.06(1)$ & $0.934(4)$ & $17.46(5)$ & $0.538(3)$ & IBA08 \\
\hline FR Ori & $0.8831653(2)$ & 38.638 & $5.8(V)$ & $\mathrm{E}$ & & & $7830^{*}$ & & & & & $0.325(2)$ & YAN14 \\
\hline V392 Ori & $0.6592821(4)$ & $40.575(7)$ & $2.4(2)(B)$ & $\mathrm{SB} 1+\mathrm{E}$ & $2.0 *(2)$ & $2.04(7)$ & $8300^{*}$ & $4.12(5)$ & $0.49(5)$ & $1.15(4)$ & $4.3(1)$ & $0.252(2)$ & NAR02a, ZHA15b \\
\hline MX Pav & $5.730835(4)$ & $13.22722(1)$ & $76.9(3)(V)$ & $\mathrm{E}$ & & & 8200 & & & & & & PIG07 \\
\hline BG Peg & $1.952404(1)$ & $25.544(1)$ & 13.1(6) (B) & $\mathrm{E}$ & $2.5^{*}$ & $2.85(3)$ & $9000^{*}$ & $3.93(1)$ & $0.55(3)$ & $2.37(3)$ & $9.76(8)$ & $0.219(1)$ & SOY09, DVO09, SOY11, LIA11, SEN14 \\
\hline
\end{tabular}


Table $\mathbf{1}$ - continued

\begin{tabular}{|c|c|c|c|c|c|c|c|c|c|c|c|c|c|}
\hline Name & $\begin{array}{l}P_{\text {orb }} \\
\text { (days) }\end{array}$ & $\begin{array}{c}f_{\text {dom }} \\
\left(\text { cycle d }^{-1}\right)\end{array}$ & $\begin{array}{c}A \\
(\mathrm{mmag})\end{array}$ & $\mathrm{ToV}$ & $\begin{array}{l}M_{\mathrm{pul}} \\
\left(\mathrm{M}_{\odot}\right) \\
\end{array}$ & $\begin{array}{l}R_{\text {pul }} \\
\left(\mathrm{R}_{\odot}\right) \\
\end{array}$ & $\begin{array}{c}T_{\text {pul }} \\
(\mathrm{K})\end{array}$ & $\begin{array}{r}\log g_{\mathrm{pul}} \\
\left(\mathrm{cm} \mathrm{s}^{-2}\right) \\
\end{array}$ & $\begin{array}{c}M_{\text {comp }} \\
\left(\mathrm{M}_{\odot}\right)\end{array}$ & $\begin{array}{c}R_{\text {comp }} \\
\left(\mathrm{R}_{\odot}\right)\end{array}$ & $\begin{array}{c}a \\
\left(\mathrm{R}_{\odot}\right) \\
\end{array}$ & $q$ & References \\
\hline AB Per & $7.16023(2)$ & 5.116 & $20.0(B)$ & $\mathrm{SB} 2+\mathrm{E}$ & 1.9 & 2.0 & $8200^{*}$ & 4.1 & 0.23 & 4.2 & 20.58 & 0.12 & $\begin{array}{l}\text { KIM03, KIM04a, BUD04, } \\
\text { KIM06, SMA14 }\end{array}$ \\
\hline IU Per & $0.8570201(1)$ & 42.103 & $20.0(B)$ & $\mathrm{E}$ & $2.2^{*}$ & 2.04 & $8450^{*}$ & 4.16 & 0.6 & 1.46 & 5.35 & $0.273(5)$ & KIM05c, SOY06b, ZHA09, KUN13 \\
\hline AO Ser & $0.8793463(3)$ & 21.505 & $20.0(B)$ & $\mathrm{E}$ & $1.82^{*}$ & 1.56 & $7800^{*}$ & 4.3 & 0.428 & 1.26 & 5.055 & $0.235(3)$ & KIM04c, YAN10, ALT12 \\
\hline UZ Sge & $2.215753(2)$ & $46.652(6)$ & $3.1(3)(B)$ & $\mathrm{E}$ & $2.1^{*}(2)$ & $1.9(2)$ & $8700^{*}$ & $4.2(1)$ & $0.29(2)$ & $2.2(2)$ & $9.8(8)$ & $0.14(1)$ & LIAN12 \\
\hline AC Tau & $2.043420(7)$ & $17.533(1)$ & $6.0(1.0)(V)$ & $\mathrm{E}$ & 1.45 & 2.30 & 7162 & 3.88 & 0.99 & 2.90 & 9.33 & 0.68 & BUD04, SOY06c, DVO09 \\
\hline IZ Tel & $4.880219(4)$ & $13.55801(2)$ & $45.9(4)(V)$ & $\mathrm{E}$ & & & & & & & & & PIG07 \\
\hline IO UMa & $5.5201878(1)$ & $22.0148(1)$ & $6.5(2)(B)$ & $\mathrm{SB} 2+\mathrm{E}$ & $2.11(7)$ & $3.00(4)$ & 7800 & $3.81(2)$ & $0.29(2)$ & $3.92(5)$ & $17.5(1)$ & $0.135(3)$ & LIA12, SOYD13 \\
\hline VV UMa & $0.6873778(4)$ & $48.8483(3)$ & $2.8(1)(V)$ & $\mathrm{SB} 1+\mathrm{E}$ & $2.5^{*}$ & 1.77 & $9500^{*}$ & 4.34 & 0.84 & 1.36 & 5.0 & $0.337(2)$ & STR50, LAZ01, KIM05a, GUN15, TAN15 \\
\hline $1200-03937339^{\text {cat } 2}$ & $1.17962(1)$ & $30.668(1)$ & 5.1(4) $(W)$ & $\mathrm{E}$ & $1.6(2)^{*}$ & $2.24(4)$ & $7250^{*}$ & $3.9(1)$ & $0.3(1)$ & $1.44(3)$ & $6.0(1)$ & $0.19(2)$ & LIA14 \\
\hline BF Vel & $0.7040277(1)$ & $44.94(2)$ & $2.6(2)(B)$ & $\mathrm{E}$ & $1.98^{*}$ & $1.77(1)$ & $8550^{*}$ & $4.23(4)$ & $0.84(8)$ & $1.481(6)$ & $4.816(6)$ & $0.424(2)$ & MAN09 \\
\hline \multicolumn{14}{|c|}{ Detached systems } \\
\hline V551 Aur & $1.173203(3)$ & $7.7270(7)$ & $19.2(3)(V)$ & $\mathrm{E}$ & & & $7000^{*}$ & & & & & $0.725(6)$ & LIU12 \\
\hline V389 Cas & $2.4948239(5)$ & $27.1(8)$ & $8.8(R)$ & $\mathrm{E}$ & & & $7673^{*}(31)$ & & & & & & KOR15 \\
\hline $\mathrm{RS} \mathrm{Cha}^{b 1, e} \mathrm{~A}$ & $1.6698822(8)$ & 21.11 & & $\mathrm{SB} 2+\mathrm{E}$ & $1.89(1)$ & $2.15(6)$ & $7638(176)$ & $4.05(6)$ & $1.87(1)$ & $2.36(6)$ & $9.20(3)$ & $0.970(7)$ & JOR75, MCI77, ALE05, \\
\hline RS Cha ${ }^{b 1, e}$ B & & 12.81 & & & $1.87(1)$ & $2.36(6)$ & $7228(166)$ & $3.96(6)$ & & & & & ВOH09, WOO13 \\
\hline CoRoT 105906206 & $3.6945708(1)$ & $9.4175(1)$ & $2.55(1)(C o R)$ & $\mathrm{SB} 2+\mathrm{E}$ & $2.25(4)$ & $4.24(2)$ & $6750(150)$ & $3.53(1)$ & $1.29(3)$ & $1.34(1)$ & $15.32(8)$ & $0.574(8)$ & SIL14 \\
\hline $\mathrm{AK} \mathrm{Crt}^{b 2}$ & $2.778758(4)$ & $14.71370(2)$ & 8-35 $(V)$ & E & & & 7800 & & & & & & PIG07 \\
\hline GK Dra ${ }^{e}$ & $9.9742(2)$ & $8.4907(1)$ & $40(V)$ & $\mathrm{SB} 2+\mathrm{E}$ & $1.8(1)$ & $2.83(5)$ & $6878(57)$ & $3.79(4)$ & $1.46(7)$ & $2.43(4)$ & $28.9(4)$ & $0.81(6)$ & DAL02, ZWI03, GRI03 \\
\hline HN Dra ${ }^{b 3, e}$ & $1.80075(3)$ & $8.5583(3)$ & $8.9(7)(B)$ & $\mathrm{SB} 2+\mathrm{EV}$ & $1.87^{*}$ & 2.87 & $6920^{*}$ & 3.84 & 1.30 & 1.42 & 9.36 & $0.690(7)$ & CHA04 \\
\hline HZ Dra & 0.77293968 & $51.068(2)$ & $4.0(4)(B)$ & $\mathrm{SB} 1+\mathrm{E}$ & $3.0(3)$ & $2.3(1)$ & $9800^{*}$ & $4.2(1)$ & $0.4(1)$ & $0.8(1)$ & $5.3(1)$ & $0.12(4)$ & LIA12 \\
\hline OO $\mathrm{Dra}^{b 4}$ & 1.2383777 & $41.867(1)$ & $4.9(3)(B)$ & $\mathrm{SB} 1+\mathrm{E}$ & $1.97(25)$ & $2.04(9)$ & $8500^{*}$ & $4.11(7)$ & $0.19(3)$ & $1.17(5)$ & $6.42(6)$ & $0.097(2)$ & DIM08a, ZHA14 \\
\hline HD $172189^{b 5, e}$ & 5.70165 & $19.5818(3)$ & & $\mathrm{SB} 2+\mathrm{E}$ & $2.06(15)$ & $4.01(9)$ & $7920(190)$ & $3.55(1)$ & $1.87(14)$ & $2.97(7)$ & $21.2(5)$ & $0.91(4)$ & MAR05, COS07, CRE09, IBA09 \\
\hline HD $220687^{b 6}$ & $1.594251(3)$ & $26.16925(4)$ & $12.8(1)(V)$ & $\mathrm{E}$ & & & $9000^{*}$ & $0.00(1)$ & 1.08 & $2.04(1)$ & $21.0(0)$ & $0.01(1)$ & PIG07 \\
\hline V644 $\operatorname{Her}^{b 7, e}$ & $11.85859(4)$ & 8.688 & $40.0(\mathrm{~V})$ & SB1 & & & & & & & & & BAR82, ROD00, DUC11 \\
\hline HIP $7666^{b 8}$ & $2.37229(8)$ & $24.465(8)$ & $1.7(V)$ & $\mathrm{E}$ & & & $8200^{*}$ & & & & & & $\mathrm{ESC} 05$ \\
\hline AI Hya ${ }^{e}$ & $8.289649(7)$ & 7.24478 & $7.6(B)$ & $\mathrm{SB} 2+\mathrm{E}$ & $1.98(4)$ & $2.77(2)$ & $7100(60)$ & $3.850(4)$ & $2.15(4)$ & $3.92(3)$ & $28.30(9)$ & $1.08(1)$ & JOR78, POP88, EKE14 \\
\hline KIC $04544587^{e}$ & $2.189097(3)$ & $48.02231(4)$ & $0.329^{k}(4)(K p)$ & $\mathrm{SB} 2+\mathrm{E}$ & $1.98(7)$ & $1.82(3)$ & $8600(100)$ & $4.241(9)$ & $1.61(6)$ & $1.58(3)$ & $10.86(5)$ & $0.813(2)$ & HAM13 \\
\hline KIC 06629588 & $2.264471(3)$ & $13.39649(1)$ & $3.05^{k}(1)(K p)$ & $\mathrm{E}$ & $1.2 *(3)$ & $1.8^{*}(7)$ & $6787^{*}(247)$ & $4.0 *(4)$ & & & & & HUB14, LIA16 \\
\hline KIC $09851944^{b 1} \mathrm{~A}$ & $2.16390189(8)$ & $10.399692(2)$ & $0.653^{k}(8)(K p)$ & $\mathrm{SB} 2+\mathrm{E}$ & $1.76(7)$ & $2.27(3)$ & $7026(100)$ & $3.96(3)$ & $1.79(7)$ & $3.19(4)$ & $10.7(1)$ & $1.01(3)$ & GUO16 \\
\hline KIC $09851944^{b 1}$ B & & & & & $1.79(7)$ & $3.19(4)$ & $6902(100)$ & $3.69(3)$ & & & & & \\
\hline KIC 10661783 & $1.231363(1)$ & 28.135 & $4.163(8)(K p)$ & $\mathrm{SB} 2+\mathrm{E}$ & $2.10(3)$ & $2.58(2)$ & $7764(49)$ & $3.938(4)$ & $0.191(3)$ & $1.12(2)$ & $6.52(6)$ & $0.091(2)$ & SOU11, LEH13 \\
\hline KIC 10686876 & $2.618427(4)$ & $21.0243(2)$ & $0.289^{k}(2)(K p)$ & E & $1.9 *(2)$ & $2.4^{*}(8)$ & $8167 *(285)$ & $3.9 *(4)$ & & & & & GIE12, HUB14, this paper \\
\hline KIC 11401845 & $2.1613(2)$ & $13-24.5$ & & E & $1.7 *(2)$ & $2.3^{*}(7)$ & $7813^{*}(273)$ & $3.9 *(4)$ & & & & & HUB14, GAU14 \\
\hline V577 $\mathrm{Oph}^{a 4, e}$ & $6.079089(5)$ & $14.3906(1)$ & $28.9(\mathrm{~V})$ & $\mathrm{SB} 2+\mathrm{E}$ & $1.60 *(1)$ & & & & $1.50(2)$ & & & $0.939(6)$ & VOL90, DIE93, ZHO01a, VOL10, CRE10 \\
\hline FL Ori & $1.550972(1)$ & 18.178 & $44.0(V)$ & $\mathrm{E}$ & 2.90 & 2.10 & 8700 & 4.3 & 1.93 & 2.17 & 9.76 & 0.67 & BUD04, ZAS11 \\
\hline XX Pyx & 1.151145 & 38.110 & $10.1(3)(B)$ & $\mathrm{SB} 1+\mathrm{EV}$ & & 2.t. & $8500^{*}$ & & 1.00 & are & o.r. & . & ARE01, HAN02, AER02 \\
\hline V5548 $\mathrm{Sgr}^{69, e}$ & 8.115800 & 8.818 & $17.1(B)$ & SB1 & $1.69^{*}$ & & $7600^{*}$ & & & & & & WAL69, HIL92, DUC11 \\
\hline $18 \mathrm{Vul}^{b 10, e}$ & $9.31408(4)$ & 8.2305 & $10.0(V)$ & $\mathrm{SB} 2$ & $2.4^{*}$ & $3.5(3)$ & $8300 *(300)$ & $3.73(7)$ & $2.2^{*}$ & $2.4(2)$ & $31.7(1)$ & $0.91(2)$ & ROD00, FEK13 \\
\hline \multicolumn{14}{|c|}{ Unclassified systems } \\
\hline KW Aurc1 & $3.7886(2)$ & $11.431(8)$ & $80.0(\mathrm{~V})$ & SB1 & $2.36(6)$ & & 7800 & $3.4^{*}$ & & & & & HUD71, MOR76, ZOR12, GAL12 \\
\hline RS Gru & 11.49425 & 6.80218 & $600.0(V)$ & SB1 & & & $7500^{*}$ & & & & & & NAM76, DER09, GAR12 \\
\hline HD $061199^{c 2}$ & $3.5744(3)$ & $25.256(1)$ & $1.46(5)(V)$ & SB2 & $2.1^{*}$ & $2.4^{*}$ & $8000(200)$ & $4.0(5)^{*}$ & 2.03 & & & $0.968(4)$ & HAR08 \\
\hline HD $207651^{c 3}$ & $1.470739(6)$ & $15.43(1)$ & $21(2)(B)$ & $\mathrm{SB} 2+\mathrm{EV}$ & $1.9^{*}$ & & 8200 & & & & & & HEN04, FEK15 \\
\hline TT Hor & $2.608215(3)$ & $38.7(4)$ & $20(4)(V)$ & $\mathrm{E}$ & & & & & & & & & MOR13 \\
\hline KIC 04570326 & $1.121553(1)$ & $8.5-20$ & & EV & $1.5 *(2)$ & $2.1^{*}(6)$ & $7000 *(140)$ & $4.0^{*}(2)$ & & & & & HUB14, GAU14 \\
\hline KIC 04739791 & $0.8989(2)$ & $20.7389(1)$ & $1.97(5)(K p)$ & $\mathrm{E}$ & $1.75 *(8)$ & $2.46 *(9)$ & $7761 *(271)$ & $3.9 *(4)$ & & & & & HUB14, LEE16b \\
\hline KIC 05197256 & $6.9634(2)$ & $9.847(2)$ & & $\mathrm{E}$ & $1.9 *(2)$ & $2.8 *(6)$ & $7832 *(274)$ & $3.9 *(4)$ & & & & & HUB14, TUR15 \\
\hline KIC 05783368 & $1.8639(5)$ & $8.5-22$ & & $\mathrm{E}$ & $1.8^{*}(2)$ & $2.8^{*}(5)$ & $8133^{*}(284)$ & $3.8^{*}(4)$ & & & & & HUB14, GAU14 \\
\hline KIC 05872506 & $2.126109(6)$ & $8.5-24.5$ & & $\mathrm{EV}$ & $1.6 *(3)$ & $2.5 *(7)$ & $7794(244)^{*}$ & $3.9 *(3)$ & & & & & HUB14, GAU14 \\
\hline KIC 06541245 & 1.6 & $19-24.5$ & & EV & & & $6545^{*}(229)$ & $4.2 *(4)$ & & & & & HUB14, GAU14 \\
\hline KIC $11973705^{c 4}$ & $6.77214(2)$ & 28.260 & & $\mathrm{SB} 2+\mathrm{EV}$ & $1.6(3)$ & $2.0^{*}$ & $7404^{*}$ & $4.039^{*}$ & & & & & LEH11, BAL11, CAT11 \\
\hline $\mathrm{DG}_{\mathrm{Leo}}{ }^{c 5}$ & $4.146751(5)$ & 11.994 & $6.2(B)$ & $\mathrm{SB} 2+\mathrm{EV}$ & $2.0(2)$ & $2.96(20)$ & $7470(220)$ & $3.8(1)$ & $2.0(2)$ & $2.94(20)$ & $17.65(1)$ & $1.000(1)$ & LAM05, FRE05 \\
\hline CQ Lyn & $12.50736(8)$ & 8.8673 & $40.0(V)$ & SB2 & $1.71(6)$ & $2.6(3)$ & $6760(50)$ & $3.85(8)$ & $1.07(4)$ & $1.0(1)$ & $32.7(1)$ & $0.626(4)$ & CAR02 \\
\hline
\end{tabular}

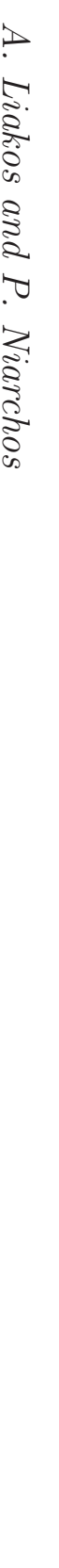


Table 1 - continued

\begin{tabular}{|c|c|c|c|c|c|c|c|c|c|c|c|c|c|}
\hline Name & $\begin{array}{c}P_{\text {orb }} \\
\text { (days) }\end{array}$ & $\begin{array}{c}f_{\text {dom }} \\
\left(\text { cycle d }^{-1}\right)\end{array}$ & $\begin{array}{c}A \\
(\mathrm{mmag})\end{array}$ & $\mathrm{ToV}$ & $\begin{array}{l}M_{\text {pul }} \\
\left(\mathrm{M}_{\odot}\right)\end{array}$ & $\begin{array}{l}R_{\text {pul }} \\
\left(\mathrm{R}_{\odot}\right)\end{array}$ & $\begin{array}{c}T_{\text {pul }} \\
(\mathrm{K})\end{array}$ & $\begin{array}{l}\log g_{\mathrm{pul}} \\
\left(\mathrm{cm} \mathrm{s}^{-2}\right)\end{array}$ & $\begin{array}{c}M_{\text {comp }} \\
\left(\mathrm{M}_{\odot}\right)\end{array}$ & $\begin{array}{c}R_{\text {comp }} \\
\left(\mathrm{R}_{\odot}\right)\end{array}$ & $\begin{array}{c}a \\
\left(\mathrm{R}_{\odot}\right)\end{array}$ & $q$ & References \\
\hline RZ Mic & $3.9830406(7)$ & $13.52(4)$ & $19.0(6)(V)$ & $\mathrm{E}$ & & & & & & & & & STR16 \\
\hline V937 Mon ${ }^{c 6}$ & $3.20865(1)$ & $9.05142(1)$ & $41.7(1)(V)$ & E & & & & & & & & & PIG07 \\
\hline V1004 Ori $^{c 7}$ & 2.74050 & $14.9(2)$ & $12.3(B)$ & SB1 & $2.15^{*}(2)$ & $2.78^{*}(21)$ & $7706^{*}(30)$ & $3.71 *(6)$ & & & & & POU04, DEB09 \\
\hline GX Pegc8 & 2.3410 & 17.857 & & SB1 & & & & & & & & & MIC93, POU04, АBT04 \\
\hline HM Pup & $2.589685(3)$ & $32(1)$ & $10(4)(V)$ & $\mathrm{E}$ & & & $7800^{*}$ & & & & & & MOR13 \\
\hline LY Pup & 2.887907 & $27.2(1)$ & $20(1)(V)$ & $\mathrm{E}$ & & & & & & & & & STR16 \\
\hline V632 Sco & 3.2204 & $25.6(1)$ & $6(1)(V)$ & $\mathrm{E}$ & 1.99 & 1.67 & 6170 & 4.29 & 0.96 & 0.25 & 8.28 & 0.48 & BRA80, ALF12, STR16 \\
\hline V638 Sco & 2.3582287 & $15.2(2)$ & $5(1)(V)$ & $\mathrm{E}$ & 2.60 & 3.19 & 8900 & 3.85 & 1.25 & 0.58 & 11.69 & 0.48 & BRA80, ALF12, STR16 \\
\hline V483 $\mathrm{Tau}^{c 9}$ & $2.486(2)$ & 17.25689 & $2.07(V)$ & SB1 & & & & & & & & & KAY99, PAPA00 \\
\hline V775 $\mathrm{Tau}^{c 10}$ & $2.1436117(8)$ & 13.0364 & $6.01(u)$ & SB1 & & & & & & & & & ZHI00a, GRI12 \\
\hline V353 $\mathrm{Tel}^{c 11}$ & 3.24886 & 23.0838 & $15.3(V)$ & EV & & & & & & & & & HANS02 \\
\hline$\theta$ Tuc & $7.1036(5)$ & 20.28061 & $18.5(2)(b)$ & SB2 & $2.1^{*}(1)$ & & $7575^{*}$ & & & & & $0.090(2)$ & MEY98, TEM00, PAP00 \\
\hline $0975-17281677^{\text {cat } 2}$ & $3.0155(8)$ & $18.702(1)$ & $6.5(3)(W)$ & $\mathrm{E}$ & & & & & & & & & LIA14 \\
\hline AW Vel & $1.992475(2)$ & $15.2(3)$ & $58(1)(V)$ & $\mathrm{E}$ & & & $7800^{*}$ & & & & & & MOR13 \\
\hline
\end{tabular}

*assumed, ${ }^{\text {cat } 1} 1$ SWASP catalogue, ${ }^{\text {cat } 2}$ USNO A2.0 catalogue,${ }^{k} \times 10^{-3}$ in relative flux units, ${ }^{e}$ eccentric orbit, ${ }^{a 1}$ UNSW-V-500, ${ }^{a 2} \mathrm{KIC} 10581918,{ }^{a 3} \mathrm{BD}+65$ 1939, ${ }^{a 4}$ triple system, ${ }^{b 1}$ both components (referred as A and B) are pulsators, ${ }^{b 2} \mathrm{HD} 099612,{ }^{b 3} \mathrm{HD} 173977,{ }^{b 4} \mathrm{GSC} 4550-1408,{ }^{b 5} \mathrm{BD}+053864,{ }^{66} \mathrm{BD}-126485,{ }^{b 7} \mathrm{HD} 152830,{ }^{b 8} \mathrm{HD} 232486,{ }^{b 9} \mathrm{~h} 01 \mathrm{Sgr}, \mathrm{HD} 184552$, ${ }^{b 10} \mathrm{HD} 191747,{ }^{c 1} 14$ Aur, HR 1706, Multiple system ${ }^{c 2} \mathrm{BD}+05$ 1734, triple system, ${ }^{c 3} \mathrm{HIP} 107786$, triple system, ${ }^{c 4} \mathrm{SPB}$ star $+\delta$ Sct or normal star + hybrid, ${ }^{c 5}$ triple system, ${ }^{c 6} \mathrm{HD} 062571$, ${ }^{c 7} 59$ Ori, HD 40372, ${ }^{c 8}$ HIP 111191 , HR 8584, ${ }^{c 9} 57$ Tau, ${ }^{c 10} 60$ Tau, HD $27628,{ }^{c 11}$ HD 173794

ABE07-Abedi \& Riazi (2007), ABT04-Abt \& Boonyarak (2004), AER02-Aerts et al. (2002), ALE05-Alecian et al. (2005), ALF12-Alfonso-Garzón et al. (2012), ALF14Alfonso-Garzón et al. (2014), ALT12-Alton \& Prša (2012), ANG06-Angione \& Sievers (2006), ARE01-Arentoft et al. (2001), BAL11-Balona et al. (2011), BAR98-Barblan et al. (1998), BAR82-Bardin \& Imbert (1982), BOH09-Böhm et al. (2009), BOZ13-Božić et al. (2013), BRA80-Brancewicz \& Dworak (1980), BUD04-Budding et al. (2004), BUD11Budding \& Butland (2011), CAR02-Carrier et al. (2002), CAT11-Catanzaro et al. (2011), CAT04-Caton (2004), CHA04-Chapellier et al. (2004), CHR07-Christiansen et al. (2007), COS07-Costa et al. (2007), CRE09-Creevey et al. (2009), CRE10-Creevey et al. (2010), DAL13-Dal \& Sipahi (2013), DAL02-Dallaporta et al. (2002), DEB09-Deb et al. (2009), DER09Derekas et al. (2009), DIE93-Diethelm (1993), DIM08a-Dimitrov et al. (2008a), DIM08b-Dimitrov et al. (2008b), DIM09a-Dimitrov et al. (2009a), DIM09b-Dimitrov et al. (2009b), DIM10-Dimitrov et al. (2010), DOG15-Doğruel \& Gürol (2015), DUC11-Ducati et al. (2011), DVO09-Dvorak (2009), EKE14-Eker et al. (2014), ESC05-Escolà-Sirisi et al. (2005), FEK13-Fekel et al. (2013), FEK15-Fekel (2015), FRE05-Frémat et al. (2005), GAL12-Galeev et al. (2012), GAR12-García (2012), GAU14-Gaulme \& Guzik (2014), GIE12-Gies et al. (2012), GOL07-Golovin \& Pavlenko (2007), GRI03-Griffin \& Boffin (2003), GRI12-Griffin (2012), GUN15-Gunsriwiwat \& Mkrtichian (2015), GUO16-Guo et al. (2016), HAM13Hambleton et al. (2013), HAM10-Hambsch et al. (2010), HAN02-Handler (2002), HANS02-Handler \& Shobbrook (2002), HAN14-Hanna (2014), HAR08-Hareter et al. (2008), HEN04Henry et al (2004), HLL92-Hildebrandt (1992), HON15-Hong et al. (2015), HOS14-Hosseinzadeh et al. (2014), HUB14-Huber et al. (2014), HUD71-Hudson et al. (1971), IBA08İbanoğlu et al. (2008), IBA09-İbanoğlu et al. (2009), JOR75-Jørgensen (1975), JOR78-Jørgensen \& Gronbech (1978), KAY99-Kaye (1999), KHA12-Khaliullin \& Khaliullina (2012), KIM02-Kim et al. (2002), KIM03-Kim et al. (2003), KIM04a-Kim et al. (2004a), KIM04b-Kim et al. (2004b), KIM04c-Kim et al. (2004c), KIM05a-Kim et al. (2005a), KIM05bKim et al. (2005b), KIM05c-Kim et al. (2005c), KIM05d-Kim et al. (2005d), KIM06-Kim et al. (2006), KIM10-Kim et al. (2010a), KOO16-Koo et al. (2016), KOR15-Korda et al. (2015), KUN13-Kundra et al. (2013), LAM04-Lampens et al. (2004), LAM05-Lampens et al. (2005), LAM11-Lampens et al. (2011), LAZ01-Lázaro et al. (2001), LEE07-Lee et al. (2007), LEE16a-Lee et al. (2016b), LEE16b-Lee et al. (2016a), LEH11-Lehmann et al. (2011), LEH13-Lehmann et al. (2013), LIA08-Liakos et al. (2008), LIA09-Liakos \& Niarchos (2009), LIA11-Liakos \& Niarchos (2011), LIA12-Liakos et al. (2012), LIAN12-Liakos \& Niarchos (2012), LIA13a-Liakos \& Niarchos (2013a), LIA13b-Liakos \& Niarchos (2013b), LIA14-Liakos \& Cagaš (2014), LIA16-Liakos \& Niarchos (2016), LIU12-Liu et al. (2012), LUC71-Lucy \& Sweeney (1971), MAN09-Manimanis et al. (2009), MAN16-Manzoori \& Salar (2016), MAR05-Martín-Ruiz et al. (2005), MCI77-McInally \& Austin (1977), MEY98-De Mey et al. (1998), MIC93-Michel et al. (1993), MKR00-Mkrtichian \& Gamarova (2000), MKR04-Mkrtichian et al. (2004), MOR76-Morguleff et al. (1976), MOR13-Moriarty et al. (2013), NAM76-McNamara \& Feltz (1976), NAR02a-Narusawa et al. (2002a), NAR13Narusawa (2013), NOR16-Norton et al. (2016), OHS01-Ohshima et al. (2001), PAP00-Paparó \& Sterken (2000), PAPA00-Paparó et al. (2000), PET12-Peters \& Wilson (2012), PIG07Pigulski \& Michalska (2007), POP11-Pop et al. (2011), POP88-Popper (1988), POU04-Pourbaix et al. (2004), ROD00-Rodríguez et al. (2000), ROD04-Rodríguez et al. (2004), ROD10Rodríguez et al. (2010), SEN08-Senyüz \& Soydugan (2008), SEN14-Senyüz \& Soydugan (2014), SIL14-Da Silva et al. (2014), SMA14-Smalley et al. (2014), SOU11-Southworth et al. (2011), SOY06a-Soydugan et al. (2006a), SOY06b-Soydugan et al. (2006b), SOY06c-Soydugan et al. (2006c), SOY07-Soydugan \& Soydugan (2007), SOY08-Soydugan et al. (2008), SOY09-Soydugan et al. (2009), SOY10-Soydugan et al. (2010), SOY11-Soydugan et al. (2011), SOY13-Soydugan \& Kaçar (2013), SOYD13-Soydugan et al. (2013), SOY16Soydugan et al. (2016), STR16-Streamer et al. (2016), SUM07-Sumter \& Beaky (2007), TAN15-Tanriver (2015), TEM00-Templeton et al. (2000), TKA09-Tkachenko et al. (2009), TKA10-Tkachenko et al. (2010), TUR08-Turcu et al. (2008), TUR11-Turcu et al. (2011), TUR15-Turner \& Holaday (2015), VOL90-Volkov (1990), VOL10-Volkov \& Volkova (2010), VYA89-Vyas \& Abhyankar (1989), WAL69-Walker \& Jones (1969), WOO13-Woollands et al. (2013), YAN10-Yang et al. (2010), YAN14-Yang et al. (2014), ZAS11-Zasche (2011), ZEJ10-Zejda et al. (2010), ZHA09-Zhang et al. (2009), ZHA14-Zhang et al. (2014), ZHA15a-Zhang et al. (2015a), ZHA15b-Zhang et al. (2015b), ZHI00a-Zhiping (2000a), ZHO01aZhou (2001a), ZOL16-Zola et al. (2016), ZOR12-Zorec \& Royer (2012), ZWI03-Zwitter et al. (2003) 
Table 2. List of detached binaries with $P_{\text {orb }}>13$ days containing a $\delta$ Sct component.

\begin{tabular}{|c|c|c|c|c|c|c|c|c|c|c|c|c|c|}
\hline Name & $\begin{array}{c}P_{\text {orb }} \\
\text { (days) }\end{array}$ & $\begin{array}{c}f_{\text {dom }} \\
\left(\text { cycle d }^{-1}\right)\end{array}$ & $\begin{array}{c}A \\
(\mathrm{mmag})\end{array}$ & $\mathrm{ToV}$ & $\begin{array}{l}M_{\text {pul }} \\
\left(\mathrm{M}_{\odot}\right)\end{array}$ & $\begin{array}{l}R_{\text {pul }} \\
\left(\mathrm{R}_{\odot}\right)\end{array}$ & $\begin{array}{l}T_{\text {pul }} \\
(\mathrm{K})\end{array}$ & $\begin{array}{l}\log g_{\text {pul }} \\
\left(\mathrm{cm} \mathrm{s}^{-2}\right)\end{array}$ & $\begin{array}{c}M_{\text {comp }} \\
\left(\mathrm{M}_{\odot}\right)\end{array}$ & $\begin{array}{c}R_{\text {comp }} \\
\left(\mathrm{R}_{\odot}\right)\end{array}$ & $\begin{array}{c}a \\
\left(\mathrm{R}_{\odot}\right) \\
\end{array}$ & $q$ & References \\
\hline BS Aqre & 11578 & $5.055(99)$ & $184(V)$ & $\mathrm{O}-\mathrm{C}$ & & & & & & & & & YAN93, ROD98a, FU99, FU00 \\
\hline CY Aqre ${ }^{e}$ & $18956(37)$ & $16.38314(1)$ & $710(V)$ & $\mathrm{O}-\mathrm{C}$ & & & & & & & & & FU00, FU03, DER09, STE12 \\
\hline$\kappa^{2}$ Boo & $2437977(88022)$ & 15.434 & $7.8(V)$ & Vis & $2.12^{*}$ & $2.78^{*}$ & $7875^{*}$ & 3.88 & & & & & FRA95, MAL12 \\
\hline $\mathrm{AI} C \mathrm{Cn}^{d 1, e}$ & $124.44(3)$ & 8.595 & $10.6(1)(y)$ & SB1 & & & $6875(120)$ & $3.3(4)$ & & & & & BRE08, SCH14 \\
\hline $\mathrm{AD} \mathrm{CMi}{ }^{e}$ & 15661(303) & $8.131768(2)$ & $143(1)(V)$ & $\mathrm{O}-\mathrm{C}$ & & & & & & & & & FU00, KHO07, HUR07, DER09 \\
\hline$\epsilon$ Cep & 6209 & 24.2471 & $7.9(B)$ & Vis & $1.8(2)$ & 2.0 & 7350 & 4.09 & & & & & $\begin{array}{l}\text { LOP79, HAR84, BAA93, } \\
\text { MAW11 }\end{array}$ \\
\hline $\begin{array}{l}\delta \operatorname{Del}^{d 2, e} \mathrm{~A} \\
\delta \operatorname{Del}^{d 2, e} \mathrm{~B}\end{array}$ & 40.58 & $\begin{array}{l}6.3291 \\
7.4627\end{array}$ & $70(\mathrm{~V})$ & SB2 & & & $7334^{*}$ & & & & & & $\begin{array}{l}\text { REI76, BAA93, ROD00, } \\
\text { PRU11 }\end{array}$ \\
\hline OR Dra ${ }^{d 3, e}$ & 49271 & $8.86297(3)$ & $11.0(7)(B)$ & Vis & & & $7000^{*}$ & & & & & & HEN01, MAL12 \\
\hline HD $051844^{d 4, e}$ & $33.498(2)$ & $12.21284(4)$ & $2.26(2)(W)$ & $\mathrm{SB} 2+\mathrm{EV}$ & $1.97(19)$ & $3.52(17)$ & $7300(200)$ & $3.65(1)$ & $2.01(20)$ & $3.7(2)$ & $69.4(5)$ & $0.99(1)$ & HAR14 \\
\hline DY $\operatorname{Her}^{d 5}$ & $15720(2620)$ & $6.72806(1)$ & $490(V)$ & $\mathrm{O}-\mathrm{C}$ & & $2.8^{*}(2)$ & & & & & & & YAN93, MIL94, POC00, POJ02 \\
\hline KZ Hya & $9788(73)$ & $16.80378(1)$ & $192(R)$ & $\mathrm{O}-\mathrm{C}$ & & & $7300 *(530)$ & $4.0^{*}(2)$ & & & & & ROD00, FU08 \\
\hline KIC $03858884^{d 6, e}$ & $25.9519(1)$ & $7.2306(1)$ & $10.2(2)(K p)$ & $\mathrm{SB} 2+\mathrm{E}$ & $1.88(3)$ & $3.45(1)$ & $6800(70)$ & $3.63(1)$ & $1.86(4)$ & $3.05(1)$ & $57.2(2)$ & $0.988(2)$ & CAT10, LEE12, MAC14 \\
\hline KIC $04150611^{d 7}$ & $94.1982(7)$ & $20.24360(3)$ & $1.610(7)(K p)$ & $\mathrm{E}$ & & & $6623^{*}$ & $4.1^{*}(4)$ & & & & & SHI12, GRE13, HUB14 \\
\hline KIC $08264492^{e}$ & $252.39(56)$ & $31.292032(2)$ & $0.893(3)(K p)$ & $\mathrm{O}-\mathrm{C}$ & $1.87^{*}(2)$ & $2.4(9)$ & $7991(279)^{*}$ & $4.0(4)^{*}$ & & & & & HUB14, SHI15, MUS15 \\
\hline $\mathrm{KIC} 08569819^{d 8, e}$ & $20.84993(3)$ & $15.857472(1)$ & $4.150(5)(K p)$ & $\mathrm{E}$ & $1.7^{*}$ & & $7100(250)$ & & 1.0 & & 44.6 & 0.588 & KUR15 \\
\hline KIC $09651065^{e}$ & $273.8(3)$ & 19.47768 & $1.931(2)(K p)$ & $\mathrm{O}-\mathrm{C}$ & $1.7^{*}(2)$ & $2.6^{*}(5)$ & $7010 *(140)$ & $3.8^{*}(2)$ & & & & & HUB14, SHI15, MUS15 \\
\hline KIC $10080943^{d 9, e} \mathrm{~A}$ & $15.3364(3)$ & $15.683330(3)$ & & $\mathrm{SB} 2+\mathrm{EV}$ & $2.0(1)$ & $2.9(1)$ & $7100(200)$ & $3.81(3)$ & $1.9(1)$ & $2.1(2)$ & 40.76 & $0.96(1)$ & SCH15, KEE15, SCH16 \\
\hline KIC $10080943^{d 9, e} \mathrm{~B}$ & & $13.947586(2)$ & & & $1.9(1)$ & $2.1(2)$ & $7480(200)$ & $4.1(1)$ & & & & & \\
\hline KIC $10990452^{e}$ & $122.11(36)$ & 17.7237 & $5.654(3)(K p)$ & $\mathrm{O}-\mathrm{C}$ & $1.7^{*}(3)$ & $2.1^{*}(4)$ & $7622 *(266)$ & $4.0^{*}(4)$ & & & & & HUB14, SHI15, MUS15 \\
\hline KIC $11754974^{e}$ & $343.27(34)$ & $16.3447449(3)$ & $51.79(5)(K p)$ & $\mathrm{SB} 1+\mathrm{O}-\mathrm{C}$ & $1.53^{*}$ & 1.76 & 7256 & 4.129 & & & & & MUR13a, MUR13b \\
\hline RY Lep & 730 & 4.4415 & $96.8(4)(I)$ & SB1 & & & & & & & & & LAN03, DER09 \\
\hline AN Lyn & $6449(94)$ & $10.175583(2)$ & $94.0(V)$ & $\mathrm{O}-\mathrm{C}$ & $2.18^{*}$ & & $7800^{*}$ & $3.8^{*}$ & & & & & $\begin{array}{l}\text { ROD97, ZHO02, HIN05, } \\
\text { LI10. PEN15 }\end{array}$ \\
\hline BE Lyn & 3475 & $10.430844(2)$ & $172.5(8)(V)$ & $\mathrm{O}-\mathrm{C}$ & & & $8700^{*}$ & $3.8^{*}$ & & & & & DER09, KIM12, PEN15 \\
\hline $\begin{array}{l}\text { DL Lyn } \\
\text { SZ Ly }\end{array}$ & $1186(5)$ & $8.2965(6)$ & $222(V)$ & $\mathrm{SB} 1+\mathrm{O}-\mathrm{C}$ & & & & & & & & & ROD00, POU04, GAZ04, LI13 \\
\hline IK Peg ${ }^{d 10}$ & 21.722 & 22.7272 & $10(V)$ & SB1 & $1.69^{*}$ & & $7600^{*}$ & & & & & & $\begin{array}{l}\text { WON93, WON94, } \\
\text { ROD00, DUC11 }\end{array}$ \\
\hline$\delta \operatorname{Ser}^{e}$ & 379119 & 6.4227 & $22.5(V)$ & Vis & & & & & & & & & ROL87, ROD00, MAL12 \\
\hline$\theta^{2} \operatorname{Tau}^{d 11, e}$ & $140.7282(9)$ & 13.220 & $4.8(1)(v)$ & SB2 & $2.86(6)$ & 4.4(1) & $7800(170)$ & $3.6(1)$ & $2.16(2)$ & $2.7(1)$ & 199(2) & $0.754(2)$ & BRE02, POR02, TOR11 \\
\hline$\rho \operatorname{Tau}^{d 12}$ & 488.5 & 14.925 & $10(V)$ & SB1 & $1.6^{*}$ & & $7300^{*}$ & & & & & & HOR79, ANT98, DUC11 \\
\hline V479 Taud13,e & 85722 & 13.1980 & $30(V)$ & Vis & & & & & & & & & DIC67, MAL12, HAR13 \\
\hline V777 Tau ${ }^{d 14, e}$ & 5200 & 5.485 & $6.0(7)(V)$ & SB1 & & & $7300^{*}$ & & & & & & KRI95, ROD00, POU04 \\
\hline GW UMa & $4821(256)$ & $4.92141(1)$ & $450(V)$ & $\mathrm{O}-\mathrm{C}$ & $1.76^{*}$ & & $7600^{*}$ & & & & & & HIN05, WAN15 \\
\hline FM Vir ${ }^{d 15, e}$ & 38.324 & 13.9119 & $20(V)$ & SB2 & $2.05^{*}$ & & & & $1.9^{*}$ & & & 0.927 & BER57, MIT79, BAR83, POU04 \\
\hline
\end{tabular}

*assumed, ${ }^{e}$ eccentric orbit, ${ }^{d 1} 4 \mathrm{CVn},{ }^{d 2} \mathrm{HD} 197461$, both components (referred as A and B) are pulsators, ${ }^{d 3} \mathrm{HD} 104288,{ }^{d 4} \mathrm{BD}-04$ 1759, ${ }^{d 5} \mathrm{ASAS} 163118+1159.8,{ }^{d 6} \mathrm{HIP} 96299, \mathrm{Hybrid}$, ${ }^{d 7} \mathrm{HD} 181469$, quintuple system, ${ }^{d 8}$ hybrid, ${ }^{d 9}$ both components (referred as A and B) are hybrid pulsators, ${ }^{d 10} \mathrm{HD} 204188$, HR $8210,{ }^{d 11} \mathrm{HD} 28319,{ }^{d 12} 86$ Tau, HD 28910, HIC 21273, ${ }^{d 13} \mathrm{HD} 24550,{ }^{d 14} 71 \mathrm{Tau},{ }^{d 15} 32 \mathrm{Vir}, \mathrm{d} 02$ Vir

ANT98-Antonello \& Pasinetti Fracassini (1998), BAA93-Baade et al. (1993), BAR83-Bartolini et al. (1983), BER57-Bertiau (1957), BRE02-Breger et al. (2002), BRE08-Breger et al. (2008), CAT10-Catanzaro et al. (2010), DER09-Derekas et al. (2009), DIC67-Dickens (1967), DUC11-Ducati et al. (2011), FRA95-Frandsen et al. (1995), FU99-Fu \& Jiang (1999),

FU00-Fu (2000), FU03-Fu \& Sterken (2003), FU08-Fu et al. (2008), GAZ04-Gazeas et al. (2004), GRE13-Gregr et al. (2013), HAR13-Hartkopf et al. (2013), HAR14-Hareter et al. (2014), HAR84-Hartkopf \& McAlister (1984), HEN01-Henry et al. (2001), HIN05-Hintz et al. (2005), HOR79-Horan (1979), HUB14-Huber et al. (2014), HUR07-Hurta et al. (2007), KEE15-Keen et al. (2015), KHO07-Khokhuntod et al. (2007), KIM12-Kim et al. (2012), KRI95-Krisciunas et al. (1995), LAN03-Laney et al. (2003), LEE12-Lee et al. (2012), LI10-Li et al. (2010), LI13-Li \& Qian (2013), LOP79-López de Coca et al. (1979), MAC14-Maceroni et al. (2014), MAL12-Malkov et al. (2012), MAW11-Mawet et al. (2011), MIL94-Milone et al. (1994), MIT79-Mitton \& Stickland (1979), MUR13a-Murphy et al. (2013a), MUR13b-Murphy et al. (2013b), MUS15-Murphy \& Shibahashi (2015), Rodríguez et al. (1997), ROD98a-Rodríguez et al. (1998a), ROD00-Rodríguez et al. (2000), ROL87-Rolland et al. (1987), SCH14-Schmid et al. (2014), SCH15-Schmid et al. (2015), SCH16-Schmid \& Aerts (2016), SHI12-Shibahashi \& Kurtz (2012), SHI15-Shibahashi et al. (2015), STE12-Sterken et al. (2012), TOR11-Torres et al. (2011), WAN15-Wang et al. (2015), WON93-Wonnacott et al. (1993), YAN93-Yang et al. (1993), ZHO02-Zhou (2002) 
Table 3. List of binaries with unspecified $P_{\text {orb }}$ containing a $\delta$ Sct component.

\begin{tabular}{|c|c|c|c|c|c|c|c|c|c|}
\hline Name & $\begin{array}{c}f_{\text {dom }} \\
\left(\text { cycle d }^{-1}\right)\end{array}$ & $\begin{array}{c}A \\
(\mathrm{mmag})\end{array}$ & $\mathrm{ToV}$ & References & Name & $\begin{array}{c}f_{\text {dom }} \\
\left(\text { cycle d }^{-1}\right)\end{array}$ & $\begin{array}{c}A \\
(\mathrm{mmag})\end{array}$ & $\mathrm{ToV}$ & References \\
\hline GN And ${ }^{1}$ & 14.4282 & $1.52(7)(V)$ & Vis & $\begin{array}{l}\text { GAR85, TUR93, } \\
\text { ROD98b, DALL02 }\end{array}$ & $\begin{array}{l}\text { HD } 050870^{19} \\
\text { HD } 064813^{5}\end{array}$ & $\begin{array}{c}17.1617 \\
20.3100(1)\end{array}$ & $\begin{array}{c}12.96(C o R) \\
2.9(1)(V)\end{array}$ & $\begin{array}{l}\text { SB2 } \\
\text { Vis }\end{array}$ & $\begin{array}{l}\text { MAN12 } \\
\text { KIMD10, HAR13 }\end{array}$ \\
\hline V361 And $^{2}$ & 7.4963 & $18.27(V)$ & Vis & FAB02, LI07 & HD $189503^{20}$ & 5.1918 & $110(V)$ & Vis & MAS01, POJ02 \\
\hline V419 And $^{3}$ & 19.4090 & $6.96(V)$ & Vis & MAR99, SMA11 & HD 218994 & 24.7 & $2.9(2)(B)$ & Vis & GON07, GON08, KUR08 \\
\hline V1208 Aql ${ }^{4}$ & $6.68072(6)$ & $18.1(V)$ & Vis & BRE76, PRO81, & HD $292962^{21}$ & $\begin{array}{c}9.18898 \\
26.323(7)\end{array}$ & $200(V)$ & Vis & MAS01, POJ02 \\
\hline$\alpha \mathrm{Aql}^{5}$ & 15.768 & & Vis & BUZ05, MAS01 & $\mathrm{BN} \mathrm{Hyi}^{23}$ & $\begin{array}{c}20.323(r) \\
15.4(2)\end{array}$ & $\begin{array}{l}2.9(1)(B) \\
20(V)\end{array}$ & $\begin{array}{l}\text { Vis } \\
\text { Vis }\end{array}$ & $\begin{array}{l}\text { MUK84, MASU1 } \\
\text { MOO83, FAB02 }\end{array}$ \\
\hline OX Aur ${ }^{6}$ & 6.8283 & $6.1(v)$ & Vis & ZHI00b, MAS01 & $\mathrm{KIC} 04840675^{5}$ & $22.06872(1)$ & $1.3715(9)$ & SB & BAL12 \\
\hline $\mathrm{BD}+184988^{7}$ & 5.779545 & $130(V)$ & Vis & FAB02, POJ02 & CC Lyn & $5.6402(4)$ & $24.5(1)(W)$ & Vis + SB1 & PRI09, CON10 \\
\hline $\mathrm{BD}-053329^{8}$ & 6.43165 & $200(V)$ & Vis & MAS01, POJ02 & XZ Men ${ }^{24}$ & 9.231 & $4.7(B)$ & Vis & KUR80, MAS01 \\
\hline$\gamma \mathrm{Boo}^{9}$ & 21.28 & & Vis & $\begin{array}{l}\text { AUV79, MAS01, } \\
\text { VEN07, DALL02 }\end{array}$ & V752 Mon ${ }^{5}$ & 4.3202 & $24.5(H p)$ & Vis & $\begin{array}{l}\text { PRI06, RUC09, } \\
\text { RUC07, DUB11 }\end{array}$ \\
\hline$\iota$ Boo & $37.750(1)$ & $3.5(V)$ & Vis & KIS95, KIS99, MAS01 & LW Mus ${ }^{25}$ & 6.5088 & $140(V)$ & Vis & MAS01, POJ02 \\
\hline $\mathrm{X} \mathrm{Cae}{ }^{10}$ & 7.394 & $37.2(V)$ & Vis & MAN00, FAB02 & CP Oct 26 & $6.67766(4)$ & $25(H p)$ & Vis & ESA97, MAS01, GON08 \\
\hline V527 $\mathrm{Car}^{11}$ & 4.680412 & $29(1)(V)$ & Vis & KOE99, FAB02 & $\mathrm{V} 2542 \mathrm{Oph}^{27}$ & $13.2624(2)$ & $11.4(7)(V+y)$ & Vis & KAY00, MAS01 \\
\hline$\beta$ Cas & $9.897396(5)$ & $15.4(7)(V)$ & Vis & RIB94, MAS01, GUI13 & $\rho$ Pup & $7.098168(1)$ & $95(V)$ & Vis & PON63, MAS01, MOO09 \\
\hline V1063 $\mathrm{Cen}^{12}$ & 20.0557 & $7.3(B)$ & Vis & FAB02, MAR02 & $\delta$ Sct & $5.16077(1)$ & $200(V)$ & Vis & STE38, MOO82, MAS01 \\
\hline V377 Cep & $13.705(1)$ & $18(R)$ & Vis & KUS95, FAB02 & $\mathrm{RX} \mathrm{Sex}{ }^{28}$ & 12.5156 & $10(V)$ & Vis & JER72, ABT81 \\
\hline HS Eri & 4.98902 & $18.3(V)$ & Vis & HOR00, KOE02 & TYC $0313-337-1^{29}$ & 6.6892 & $210(V)$ & Vis & MAS01, POJ02 \\
\hline IU $\operatorname{Eri}^{13}$ & $14.51837(6)$ & $11.8(6)(V)$ & Vis & MAS01, HANS02 & TYC $5827-482-1^{30}$ & 17.7120 & $120(V)$ & Vis & MAS01, POJ02 \\
\hline TY For ${ }^{14}$ & 10.2942 & $15(V)$ & Vis & EGG79, LAM90, FAB02 & $\mathrm{DP} \mathrm{UMa}^{31}$ & 25 & $20(V)$ & Vis & KUR78, KUR79, MAS01 \\
\hline VV For ${ }^{15}$ & 17.3310 & $10.1(2)(v)$ & Vis & STE89, MAS01 & $v \mathrm{UMa}$ & $7.54(2)$ & $71(8)(V)$ & Vis & DWO73, MAS01 \\
\hline PV Gem & 5.3173 & $50(V)$ & Vis & NIC10, HAR13 & FZ Vel & 15.3846 & $15(V)$ & Vis & EGG70, HAR13 \\
\hline DQ Gru ${ }^{16}$ & 4.679 & $15.8(B)$ & $\mathrm{Vis}+\mathrm{SB} 2$ & LAM92, TSV93, & V540 $\mathrm{Vel}^{32}$ & 5.9192 & $46(V)$ & Vis & MAS01, KHR10 \\
\hline & & & & LAM99, LAM00 & FG Vir & 12.716 & $22.1(Y)$ & Vis & ABT81, LEN08, MAS01 \\
\hline HD $011667^{17}$ & 10.9888 & $290(V)$ & Vis & MAS01, POJ02 & GG $\operatorname{Vir}^{33}$ & 17.8571 & $13(B)$ & Vis & BAR75, STE77, MAS01 \\
\hline HD $017421^{18}$ & $\begin{array}{l}10.9000 \\
12.4510\end{array}$ & $80(V)$ & Vis & FAB02, POJ02 & OV $\operatorname{Vir}^{34}$ & $31.3548(2)$ & $4.9(1)(b)$ & Vis & RODR01, MAS01 \\
\hline HD 041140 & $25.827(1)$ & $4.7(2)(V)$ & Vis & FAB02, KIMD10 & & & & & \\
\hline
\end{tabular}

${ }^{1} 28$ And, ${ }^{2} \mathrm{HD} 6859,{ }^{3} \mathrm{HD} 13079,{ }^{4} 28 \mathrm{Aql}$. HR $7331,{ }^{5}$ multiple system, ${ }^{6} 59$ Aur, ${ }^{7}$ ASAS J222629+1924.8, ${ }^{8}$ ASAS J114026-0606.2, ${ }^{9}$ triple system, ${ }^{10} \gamma^{2}$ Cae, ${ }^{11} \mathrm{HD} 95321,{ }^{12} \mathrm{HD} 129052$, ${ }^{13} \mathrm{HD} 27093,{ }^{14} \mathrm{HIP} 11644$, HR 733, HD $15634{ }^{15} \mathrm{HD} 17978,{ }^{16} \mathrm{HD} 220392,{ }^{17} \mathrm{ASAS}$ J015307-5056.5, ${ }^{18}$ ASAS J024816+2213.4, ${ }^{19} \mathrm{BD}-03$ 1642, ${ }^{20}$ ASAS J200221-4554.0, ${ }^{21}$ ASAS J065937$0047.0,{ }^{22} 2$ Hya, HR 3321, ${ }^{23} \mathrm{HR} 981,{ }^{24} \mathrm{HD} 31908,{ }^{25}$ ASAS J133845-7011.2, ${ }^{26} \mathrm{HD} 21190,{ }^{27} \mathrm{HD} 152569,{ }^{28} \mathrm{HD} 90386,{ }^{29}$ ASAS J133737+0715.9, ${ }^{30}$ ASAS J231240-1305.8, ${ }^{31} 67$ UMa HR $4594,{ }^{32} \mathrm{HD} 298732,{ }^{33} 27 \mathrm{Vir},{ }^{34} \mathrm{HD} 129231$

ABT81-Abt (1981), AUV79-Auvergne et al. (1979), BAL12-Balona et al. (2012), BAR75-Bartolini et al. (1975), BRE76-Breger et al. (1976), BUZ05-Buzasi et al. (2005), CON10Conidis et al. (2010), DALL02-Dall \& Frandsen (2002), DUB11-Dubath et al. (2011), DWO73-Dworak (1973), EGG70-Eggen (1970), EGG79-Eggen (1979), ESA97-ESA (1997), FAB02Fabricius et al. (2002), GAR85-Garrido et al. (1985), GON07-González et al. (2007), GON08-González et al. (2008), GUI13-Guiglion et al. (2013), HANS02-Handler \& Shobbrook (2002), HAR13-Hartkopf et al. (2013), HOR00-Horch et al. (2000), JER72-Jerzykiewicz (1972), KAY00-Kaye et al. (2000), KHR10-Khruslov (2010), KIMD10-Kim et al. (2010b), KIS95Kiss (1995), KIS99-Kiss et al. (1999), KOE99-Koen et al. (1999), KOE02-Koen \& Eyer (2002), KUR78-Kurtz (1978), KUR79-Kurtz (1979), KUR80-Kurtz (1980), KUR84-Kurtz (1984), KUR08-Kurtz et al. (2008), KUR15-Kurtz et al. (2015), KUS95-Kusakin \& Goranskii (1995), LAM90-Lampens \& Rufener (1990), LAM92-Lampens (1992), LAM99-Lampens et al. (1999), LAM00-Lampens et al. (2000), LEN08-Lenz et al. (2008), LI07-Li \& Yan (2007), MAN00-Mantegazza et al. (2000), MAN12-Mantegazza et al. (2012), MAR99-Martinez et al. (1999), MAR02-Martinez (2002), MAS01-Mason et al. (2001), MOO82-Moon \& Keay (1982), MOO83-Moon et al. (1983), MOO09-Moon \& van Antwerpen (2009), NIC10-Nichols et al. (2010), PON63-Ponsen (1963), POJ02-Pojmanski (2002), PRI06-Pribulla \& Rucinski (2006), PRI09-Pribulla et al. (2009), PRO81-Proust et al. (1981), RIB94-Riboni et al. (1994), ROD98b-Rodríguez et al. (1998b), RODR01-Rodríguez et al. (2001), RUC07-Rucinski et al. (2007), SMA11-Smalley et al. (2011), STE77-Sterken (1977), STE89-Sterken \& Jerzykiewicz (1989), STE38-Sterne (1938), TSV93-Tsvetkov (1993), TUR93-Turon et al. (1993), VEN07-Ventura et al. (2007), WEH95-Wehlau \& Wehlau (1995), ZHI00b-Zhiping (2000b) 


\begin{tabular}{|c|c|c|c|c|c|}
\hline Name & Comments & References & Name & Comments & References \\
\hline \multicolumn{6}{|c|}{ Confirmed binary systems but ambiguous for pulsations type/existence } \\
\hline AD Ari & EV, but weak proof for pulsations & HANS02 & KIC 06963171 & Unclear type of pulsations & BRA15 \\
\hline VW Ari & Visual triple but probably of $\lambda$ Boo type & MUR15a & KIC 08553788 & Unclear type of pulsations & GIE12 \\
\hline ASAS $073904-6037.2^{1}$ & Weak proof for pulsation type & PIG07 & KIC 08553788 & Unclear type of pulsations & GIE12 \\
\hline ASAS $110615-4224.6^{2}$ & Weak proof for pulsation type & PIG07 & KIC 08895509 & Unclear type of pulsations & BRA15 \\
\hline ASAS $234520-3100.5^{3}$ & Weak proof for pulsation type & PIG07 & KIC 11819135 & Unclear type of pulsations & BRA15 \\
\hline NR CMa & Vis. but weak proof for pulsations & ESA97, MAS01 & KIC 11867071 & Unclear type of pulsations & BRA15 \\
\hline MM Cas & Weak proof for pulsations & CHA83, CHA92 & $\beta$ Leo & Vis. but unclear type of pulsations & BAR81, MKR98, MAS01 \\
\hline V377 Cas & Vis. but weak proof for pulsations & LOW89, FAB00 & DT Lup & Weak proof for pulsations & MID11 \\
\hline$\psi$ Cen & Unclear presence and/or type of pulsations & BRU06, MAN10 & $\alpha$ Lyr & Vis. but weak proof for pulsations & ВOH12, ВОH15 \\
\hline V360 Cep & Vis. but weak proof for pulsations & MAR82, FAB00 & EY Ori & Weak proof for pulsations & ZAK98, SMA14, TUR14 \\
\hline UU Com ${ }^{4}$ & Probably it is of $\alpha^{2}$ CVn type & SAN89, SAV96, LIP08 & FO Ori & Weak proof for pulsations & TUR14 \\
\hline$\beta \mathrm{CrB}$ & Probably it is of $\alpha^{2}$ CVn type & HAT04, KUR07, BRU10 & HT Peg & Vis. but weak proof for pulsations & CON74, MAS01 \\
\hline$\gamma \mathrm{CrB}$ & Vis. but unclear type of pulsations & VET81, LEH97, MAL12 & NN Peg & Vis. but weak proof for pulsations & FAB02 \\
\hline$\tau$ Cyg & Vis. but unclear type of pulsations & MKR95, FAB02 & V368 Pup & Vis. but weak proof for pulsations & ESA97, MAS01 \\
\hline AI Dra & Weak proof for pulsations & NAR02b, KIS02, LAZ04 & V1060 Sco & Vis. but weak proof for pulsations & ESA97, MAS01 \\
\hline CC Gru & Vis. but weak proof for pulsations & ESA97, MAS01 & V1072 Sco & Vis. but weak proof for pulsations & ESA97, MAS01 \\
\hline HD 073712 & SB2 but doubtful for pulsations & MIC95, DUC11 & V1241 $\mathrm{Tau}^{6}$ & The pulsating behaviour is questionable & ARE04, PIG07, ULA14 \\
\hline HD 120635 & Vis. but weak proof for pulsations & MAS01, RIC12 & TYC 7817-75-1 & Vis. but weak proof for pulsations & MAS01, RIC12 \\
\hline V979 Her & Vis. but unclear spectral type ( $\beta$ Cep?) & MAS01, POJ02 & $\mathrm{X}$ Tri & Weak proof for pulsations & KIM03, LIA10, TUR14 \\
\hline V994 Her $^{5}$ & Quintuple system with $2 \mathrm{E}$ & DAL05, LEE08, ZAS13 & RU UMi & Weak proof for pulsations & BEL93, MAN01, ZHU06 \\
\hline KIC 04470124 & Unclear type of pulsations & BRA15 & GZ Vir & Triple system but weak proof for pulsations & PEN81, ROD88, TOK10 \\
\hline KIC 05615815 & Unclear type of pulsations & BRA15 & HX Vir ${ }^{7}$ & Vis. but weak proof for pulsations & STE86, MAS01 \\
\hline KIC 05878081 & Unclear type of pulsations & BRA15 & $\theta \operatorname{Vir}^{8}$ & Weak proof for pulsations & BEA77, ADE97 \\
\hline \multicolumn{6}{|c|}{ Confirmed $\delta$ Sct stars but ambiguous for binarity } \\
\hline V853 Cep & Weak proof for visual binarity & VAN86 & V1366 Ori ${ }^{11}$ & Weak proof for binarity & SOL03, AMA04, CAS13 \\
\hline KIC $05988140^{9}$ & Unclear for binarity. Hybrid $\gamma$ Dor $-\delta$ Sct star & LAM13 & DL UMa & Weak proof for binarity & ROD00, NIC10 \\
\hline V474 $\mathrm{Mon}^{10}$ & Weak proof for binarity & DES74, BAL01, AMA07 & & & \\
\hline \multicolumn{6}{|c|}{ Ambiguous for both binarity and pulsations type/existence } \\
\hline HV Eri & It might be of W UMa type & PRI09 & o Ser & Weak proof for SB and for pulsations & VAL72, DWO75 \\
\hline BR Hyi & It might be of W UMa type & POJ02 & V353 Vel & It might be of W UMa type & PRI03, PRI06 \\
\hline V1359 Ori & It might be of W UMa type & PRI09 & & & \\
\hline
\end{tabular}

${ }^{1} \mathrm{CPD}-600^{\circ} 871,{ }^{2} \mathrm{CPD}-41^{\circ} 5106,{ }^{3} \mathrm{CPD}-31{ }^{\circ} 6830,{ }^{4} 21 \mathrm{Com}, \mathrm{HD} 108945,{ }^{5} \mathrm{HD} 170314,{ }^{6} \mathrm{WX}$ Eri, ${ }^{7} 73 \mathrm{Vir},{ }^{8} 51 \mathrm{Vir},{ }^{9} \mathrm{HD} 188774,{ }^{10} 1 \mathrm{Mon}, \mathrm{HD} 40535,{ }^{11} \mathrm{HD} 34282$

ADE97-Adelman (1997), AMA04-Amado et al. (2004), AMA07-Amado (2007), ARE04-Arentoft et al. (2004), BAL01-Balona et al. (2001), BAR81-Bartolini et al. (1981), BEA77-Beardsley \& Zizka (1977), BEL93-Bell et al. (1993), BOH12-Böhm et al. (2012), BOH15-Böhm et al. (2015), BRA15-Bradley et al. (2015), BRU06-Bruntt et al. (2006), BRU10-Bruntt et al. (2010), CAS13-Casey et al. (2013), CHA83-Chaubey (1983), CHA92-Chaubey (1992), CON74-Le Contel et al. (1974), DAL05-Dallaporta \& Munari (2005), DES74-Desikachary (1974), DUC11-Ducati et al. (2011), DWO75-Dworak \& Zieba (1975), ESA97-ESA (1997), FAB00-Fabricius \& Makarov (2000), FAB02-Fabricius et al. (2002), GIE12-Gies et al. (2012), HANS02-Handler \& Shobbrook (2002), HAT04-Hatzes \& Mkrtichian (2004), KIM03-Kim et al. (2003), KIS02-Kiss (2002), KUR07-Kurtz et al. (2007), LAM13-Lampens et al. (2013), LAZ04-Lázaro et al. (2004), LEE08-Lee et al. (2008), LEH97-Lehmann et al. (1997), LIA10-Liakos et al. (2010), LIP08-Lipski \& Stẹpień (2008), LOW89-Lowder (1989), MAL12-Malkov et al. (2012), MAN01-Manimanis \& Niarchos (2001), MAN10-Mantegazza et al. (2010), MAR82-Margrave et al. (1982), MAS01Mason et al. (2001), MIC95-Michel et al. (1995), MID11-Middleton (2011), MKR95-Mkritichian et al. (1995), MKR98-Mkrtichian \& Yurkov (1998), MUR15a-Murphy et al. (2015a), NAR02b-Narusawa et al. (2002b), NIC10-Nichols et al. (2010), PEN81-Peniche \& Peña (1981), PIG07-Pigulski \& Michalska (2007), POJ02-Pojmanski (2002), PRI03-Pribulla et al. (2003), PRI06-Pribulla \& Rucinski (2006), PRI09-Pribulla et al. (2009), RIC12-Richards et al. (2012), ROD88-Rodríguez et al. (1988), ROD00-Rodríguez et al. (2000), SAN89Santagati et al. (1989), SAV96-Savanov et al. (1996), SMA14-Smalley et al. (2014), SOL03-Solonovich et al. (2003), STE86-Sterken \& Jerzykiewicz (1986), TOK10-Tokovinin et al. (2010), TUR14-Turner et al. (2014), ULA14-Ulaş et al. (2014), VAL72-Valtier (1972), VAN86-Vander Linden \& Sterken (1986), VET81-Veto \& Kovacs (1981), ZAK98-Zakirov (1998), ZAS13-Zasche \& Uhlař (2013), ZHU06-Zhu et al. (2006) 


\begin{tabular}{|c|c|c|c|c|c|}
\hline Name & Comments & References & Name & Comments & References \\
\hline $\mathrm{CC}$ And & No proof for binarity & EKM08 & GSC $3382-0957$ & E or an hybrid $\gamma$ Dor $-\delta$ Sct star & ZHA12 \\
\hline ET And & It is of $\alpha^{2} \mathrm{CVn}$ type $(\mathrm{B} 9 \mathrm{p}(\mathrm{Si})$ star $)$ & WEI98 & HD 086731 & No relevant references exist & \\
\hline GP And & No proof for binarity & ZHO11 & HD 094529 & No proof for pulsations & PIG07 \\
\hline V529 And ${ }^{1}$ & No proof for binarity & HEN05 & HD 188164 & It is of $\lambda$ Boo type & MUR15a \\
\hline DV Aqr & No proof for pulsations & POL10 & CN Hyi & It is of W UMa type & RUC06 \\
\hline$\zeta$ Aur & Prototype of $\zeta$ Aur type systems & EAT08 & KIC $09204718^{5}$ & E or an hybrid $\gamma$ Dor $-\delta$ Sct star & TUR13 \\
\hline $\mathrm{BR}$ Cnc & Member of OC. Not Binary* & ZHOU01 & UX Mon & It is of W Ser type & SUD11 \\
\hline EX Cnc & Member of OC. Not Binary* & ZHO01b & DE Oct & It is of W UMa type & PRI06 \\
\hline $\mathrm{HI} \mathrm{Cnc}$ & Member of OC. Not Binary* & ZEJ12 & BQ Phe & It is of W UMa type & POU04 \\
\hline VZ CVn & It is of $\gamma$ Dor type & LAT12 & V856 Sco & It is of UX Ori type & NAT97, BEN11 \\
\hline $\mathrm{AZ} \mathrm{CMi}$ & No proof for binarity & FED91 & $v$ Tau & Member of OC. Not Binary* & BOS 83 \\
\hline V410 Car & Member of OC. Not Binary* & ANT86 & V480 Tau & Member of OC. Not Binary* & KIP78 \\
\hline RX Cas & It is of W Ser type & TOD89, TAR97, PUS07 & V534 Tau & Member of OC. Not Binary* & LI04 \\
\hline V965 Cep & No proof for binarity & SOK09 & V647 Tau & Member of OC. Not Binary* & LIU99 \\
\hline AV Cet & No proof for binarity & MAN05 & V650 Tau & Member of OC. Not Binary* & FOX11 \\
\hline $\mathrm{AI} \mathrm{Com}^{2}$ & It is of $\alpha^{2}$ CVn type. Vis. $+\mathrm{SB}$ & ADE81, SAV96 & V1128 Tau & It is of W UMa type & CAL14 \\
\hline $\mathrm{TZ} \mathrm{CrB}^{3}$ & It is of RS CVn type & FRA97 & V1178 Tau & Member of OC. Not Binary* & ARE05 \\
\hline V1644 $\mathrm{Cyg}^{4}$ & It is of $\lambda$ Boo type & CAS09 & V1229 $\mathrm{Tau}^{6}$ & No proof for pulsations & GRO07 \\
\hline V2448 Cyg & Member of OC. Not Binary* & FRE01 & TYC 1845-2532-1 & Member of OC. Not Binary* & POJ02, ZEJ12 \\
\hline ZZ Cyg & No proof for pulsations & YAN15 & GU Vel & No proof for binarity & EGG08 \\
\hline V345 Gem & It is of W UMa type & PRI06 & V377 $\mathrm{Vul}^{7}$ & It is of SPB type & HUB91, DEC07, WAL12 \\
\hline
\end{tabular}

$\mathrm{SPB}=$ Slowly pulsating B-type star, $\mathrm{OC}=$ Open cluster.

The star belongs to an $\mathrm{OC}$ but it is not member of a binary system.

${ }^{1} \mathrm{HD} 8801,{ }^{2} 17 \mathrm{Com},{ }^{3} \sigma_{2} \mathrm{CrB},{ }^{4} 29 \mathrm{Cyg},{ }^{5} \mathrm{HD} 176843,{ }^{6} \mathrm{HD} 23642,{ }^{7} 3 \mathrm{Vul}, \mathrm{HD} 182255$

ADE81-Adelman (1981), ANT86-Antonello \& Mantegazza (1986), ARE05-Arentoft et al. (2005), BEN11-Benisty et al. (2011), BOS83-Bossi et al. (1983), CAL14-Calışkan et al. (2014), CAS09-Casas et al. (2009), DEC07-De Cat et al. (2007), EAT08-Eaton et al. (2008), EGG08-Eggleton \& Tokovinin (2008), EKM08-Ekmekçi \& Topal (2008), FED91-Fedotov \& Gladyr (1991), FOX11-Fox Machado et al. (2011), FRA97-Frasca et al. (1997), FRE01-Freyhammer et al. (2001), GRO07-Groenewegen et al. (2007), GUI13-Guiglion et al. (2013), HEN05Henry \& Fekel (2005), HUB91-Hube \& Aikman (1991), KIP78-Kipp et al. (1978), LAT12-Latković (2012), LEH03-Lehmann et al. (2003), LI04-Li et al. (2004), LIU99-Liu et al. (1999), MAN05-Mantegazza \& Poretti (2005), MUR15a-Murphy et al. (2015a), NAT97-Natta et al. (1997), PIG07-Pigulski \& Michalska (2007), POJ02-Pojmanski (2002), POL10-Polubek (2001), POU04-Pourbaix et al. (2004), PRI06-Pribulla \& Rucinski (2006), PUS07-Pustylnik et al. (2007), RUC06-Rucinski \& Duerbeck (2006), SAV96-Savanov et al. (1996), SOK09Sokolovsky (2009), SUD11-Sudar et al. (2011), TAR97-Taranova \& Shenavrin (1997), TOD89-Todorova \& Khruzina (1989), TUR13-Turner \& Holaday (2013), WAL12-Walczak et al. (2012), WEI98-Weiss et al. (1998), YAN15-Yang et al. (2015), ZEJ12-Zejda et al. (2012), ZHA12-Zhang et al. (2012), ZHO01b-Zhou (2001b), ZHOU01-Zhou et al. (2001), ZHO11Zhou \& Jiang (2011) 


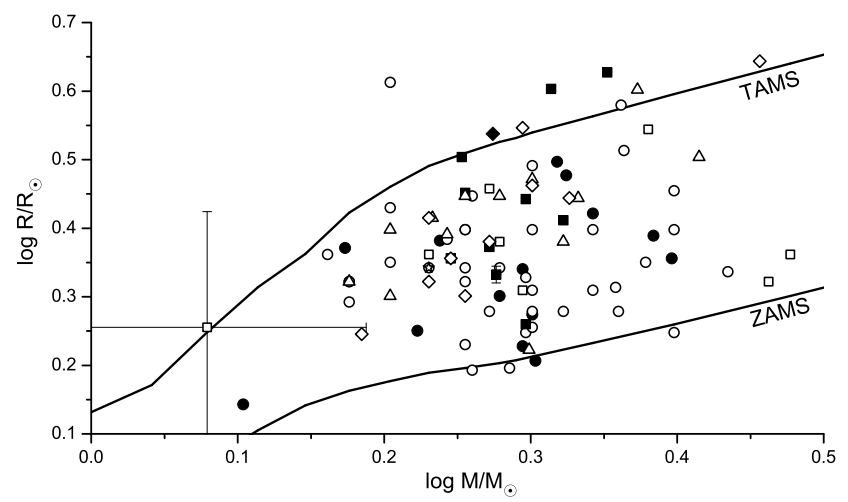

Figure 5. The distribution of binary $\delta$ Sct stars within the $M-R$ diagram. Filled symbols denote the stars with very accurate measured parameters, while the open ones those with less accurate parameters. Circles, squares, and triangles symbols correspond to the stars that belong to semi-detached, detached, and unclassified systems with $P_{\text {orb }}<13$ days, respectively. Diamond and star symbols correspond to those that belong to detached and unclassified systems with $P_{\text {orb }}>13$ days, respectively. To avoid any clutter and make the figures easier to read, only the largest and the smallest error bars that delimit the range of uncertainties are plotted.

In Fig. 5 the location of the binary $\delta$ Sct stars within the Mass-Radius $(M-R)$ diagram is shown. Zero and Terminal Age (ZAMS and TAMS, respectively) Main Sequence lines (for solar metallicity composition, i.e. $Z=0.019$ ) were taken from Girardi et al. (2000). Stars with accurately calculated parameters are considered those which are double-line spectroscopic eclipsing binaries, while all of the parameters of the rest of the systems are considered to be of lower accuracy. In this plot $84 \delta$ Sct stars that belong to binaries with $P_{\text {orb }}<13$ days (for sample details see Fig. 2) and 12 cases that belong to systems with $P_{\text {orb }}>13$ days are shown.

The distribution of the binary $\delta$ Sct stars is plotted within the Hertzsprung-Russell $(H-R)$ in Fig. 6. ZAMS and TAMS lines as well as the evolutionary tracks for three different initial mass values and for solar metallicity composition were taken from Girardi et al. (2000). The boundaries $(\mathrm{B}=$ Blue, $\mathrm{R}=\mathrm{Red})$ of the instability strip (IS) were taken from Soydugan et al. (2006b). The sample is the same with that of Fig. 5, except for one case (V577 Oph) whose temperature has not been found yet.

The locations of the binary $\delta$ Sct stars within the $T_{\text {eff }}-$ $\log g$ diagram are given in Fig. 7. The sample consists of 87 $\delta$ Sct stars in binaries with $P_{\text {orb }}<13$ days and 20 more stars in systems with $P_{\text {orb }}>13$ days. The instability strip lines were taken from Murphy et al. (2015b) and correspond to modes with $l \leqslant 2$, while ZAMS and TAMS lines, likely in Figs 5-6, were taken from Girardi et al. (2000).

Fig. 8 contains the distribution of binary $\delta$ Sct stars according to their $f_{\text {dom }}$ over the $M-R$ diagram. The sample is the same as in Fig. 5. Horizontal plane corresponds to the $M-R$ diagram, while the vertical axis to the $f_{\text {dom }}$.

\section{CORRELATIONS}

Following previous works (e.g. Soydugan et al. 2006b; Liakos et al. 2012; Zhang et al. 2013) correlations between

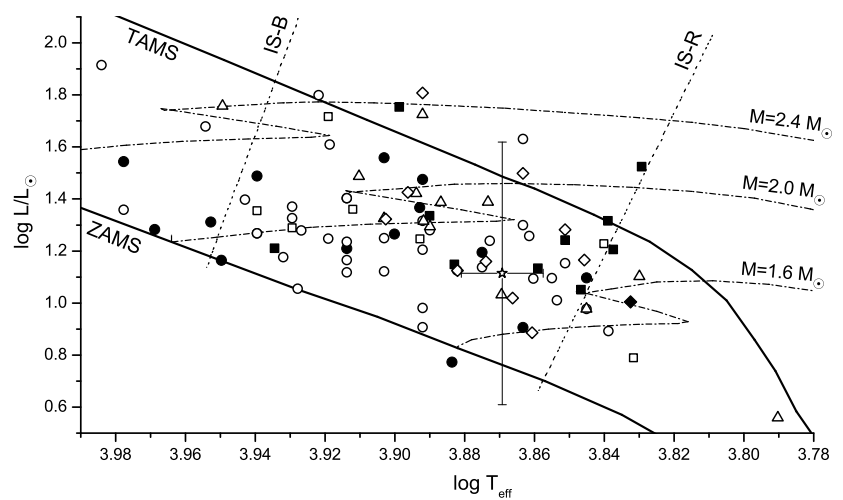

Figure 6. The distribution of binary $\delta$ Sct stars within the $H-R$ diagram. Dotted lines represent the boundaries of the instability strip (Blue and Red edges) and the dash-dotted lines the evolutionary tracks for three different initial mass values. Symbols, lines, and error bars have the same meaning as in Fig. 5.

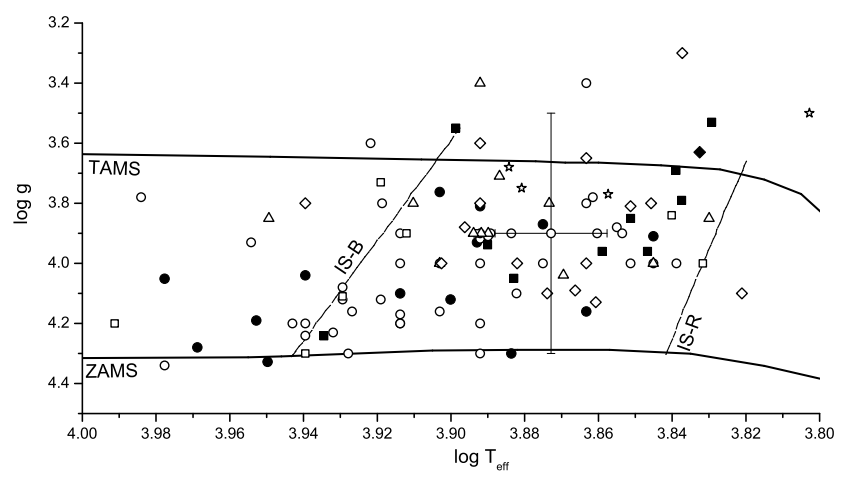

Figure 7. The distribution of binary $\delta$ Sct stars within the $T_{\text {eff }}-\log g$ diagram. Symbols, lines, and error bars have the same meaning as in Figs. 5 and 6.

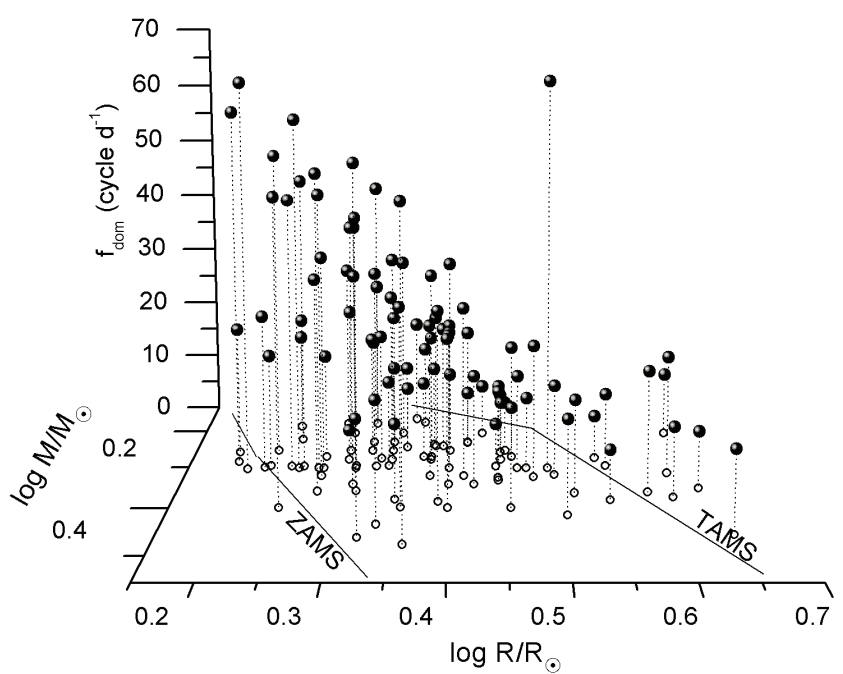

Figure 8. The distribution of binary $\delta$ Sct stars according to their $f_{\text {dom }}$ over the $M-R$ diagram. Dash lines indicate the projection of each point on the $M-R$ diagram. 


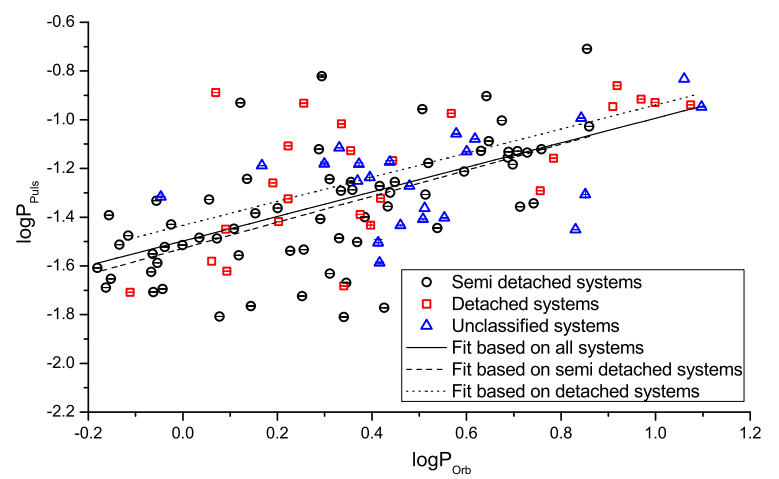

Figure 9. The correlation between $\log P_{\text {orb }}-\log P_{\text {pul }}$ of binary $\delta$ Sct stars in systems with $P_{\text {orb }}<13$ days. Three different linear fits (solid, dashed, and dashed-dotted lines) on different data samples (all, semi detached, and detached systems) are shown.

fundamental stellar parameters are presented here for the systems with $P_{\text {orb }}<13$ days (i.e. the first subgroup of Fig. 4).

The first correlation concerns the connection between $P_{\text {orb }}-P_{\text {pul }}$, which shows the dependence of the pulsations of the $\delta$ Sct star on the orbital period value of the system. In Figs 9 and 10 the linear fits using logarithmic (Fig. 9) and decimal (Fig. 10) values of three different data samples are shown. The first fit was made on all data points (114), the second one on points that correspond to semi-detached systems (66), and the third one on those corresponding to detached systems (25). Plots and correlations are made for both logarithmic and decimal values so that can be directly compared with those of previous studies (Soydugan et al. 2006b; Liakos et al. 2012; Zhang et al. 2013). The correlations between $P_{\text {orb }}-P_{\text {pul }}$ for each sample along with the respective correlation coefficient $r$ are presented below:

for $\delta$ Sct stars in Semi Detached binaries:

$$
\begin{gathered}
\log P_{\mathrm{pul}}=-1.53(3)+0.54(8) \log P_{\mathrm{orb}}, \text { with } r=0.62 \\
P_{\mathrm{pul}}=0.020(6)+0.012(2) P_{\mathrm{orb}}, \text { with } r=0.61
\end{gathered}
$$

for $\delta$ Sct stars in Detached binaries:

$$
\begin{gathered}
\log P_{\mathrm{pul}}=-1.43(7)+0.49(13) \log P_{\mathrm{orb}}, \text { with } r=0.60 \\
P_{\mathrm{pul}}=0.043(9)+0.007(2) P_{\mathrm{orb}}, \text { with } r=0.61
\end{gathered}
$$

for $\delta$ Sct stars in all known close binaries (Detached, Semidetached, unclassified):

$$
\begin{gathered}
\log P_{\mathrm{pul}}=-1.50(3)+0.50(6) \log P_{\mathrm{orb}}, \text { with } r=0.62 \\
P_{\mathrm{pul}}=0.031(4)+0.009(1) P_{\mathrm{orb}}, \text { with } r=0.62
\end{gathered}
$$

The second correlation shows the dependence between the pulsation periods of the binary $\delta$ Sct stars and their evolutionary stages. Fig. 11 shows the fit on the $\log g-\log P_{\text {pul }}$ values of the binary $\delta$ Sct stars. It should be noted that the $\log g$ values of the pulsators, when not directly given in the literature, were calculated using the following formula:

$$
\log g_{\mathrm{pul}}=4.438+\log M_{\mathrm{pul}}-2 \log R_{\mathrm{pul}}
$$

where 4.438 is the $\log g$ value of the Sun (in $\mathrm{cm} \mathrm{s}^{-2}$ ), while

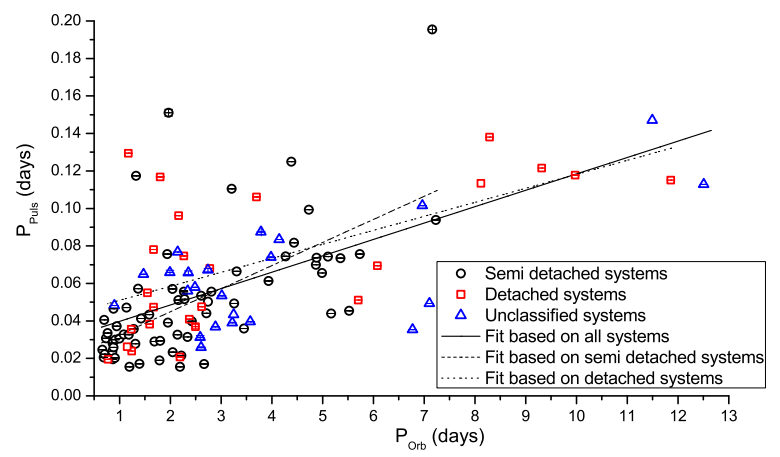

Figure 10. The same as in Fig. 9, but for the decimal $P_{\text {orb }}-P_{\text {pul }}$ values.

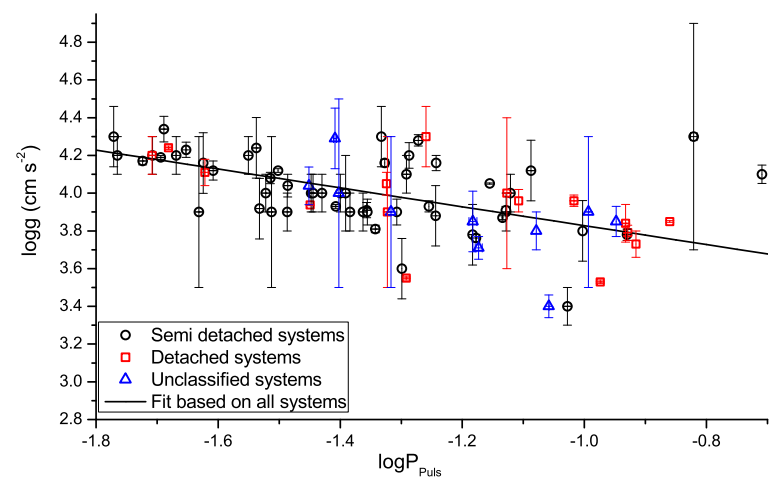

Figure 11. The correlation between $\log P_{\mathrm{pul}}-\log g$ of binary $\delta$ Sct stars in systems with $P_{\text {orb }}<13$ days.

the mass $\left(M_{\text {pul }}\right)$ and the radius $\left(R_{\text {pul }}\right)$ of the star are given in solar units.

The errors of $\log g$ values for 21 out of 82 binary $\delta$ Sct stars are not given in the literature. Therefore, their error values were assigned the average values of the errors of the stars of other systems whose absolute parameters were calculated based on the same available information. The reliability and the accuracy of the $\log g$ value and its error are directly connected to the available information for their derivation (e.g. RVs+light curves model, or light curves model only). In particular, we found 0.05 as an average $\log g_{\mathrm{pul}}$ error value for SB2+E, 0.16 for E, 0.06 for SB1, 0.1 for SB2+EV, and 0.07 for SB1+E systems. The whole sample consists of 82 stars, whose details are given in Table 1 and their demographics are shown in Fig. 2. Based on the above assumptions, the following correlation is derived:

$$
\log g=3.33(1)-0.50(1) \log P_{\mathrm{pul}} \text {, with } r=0.68
$$

The gravitational force per gram matter of the pulsator's surface $F / M_{\text {pul }}$ expresses the influence of the companion's gravity to the $P_{\mathrm{pul}}$ of the $\delta$ Sct star. The mass transfer is not taken into account in this correlation, although it probably plays a crucial role in the $P_{\text {pul }}$ modulation. However, so far, it is the only way to quantify the influence of the binarity on the dominant oscillation frequency. For the calculation of the $F / M_{\text {pul }}$ the formalism of Cakırl \& İbanoğlu (2016, eq. 10) was used. The $F / M_{\text {pul }}$ is derived for each system in cgs units (i.e. dyn $\mathrm{gr}^{-1}$ ), while the $P_{\text {pul }}$ is expressed

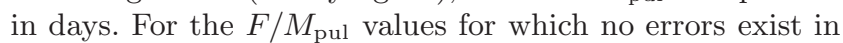




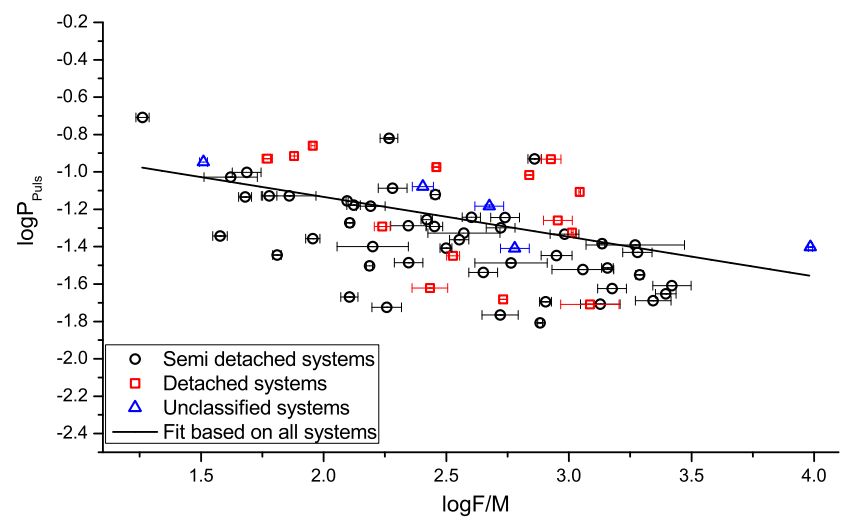

Figure 12. The correlation between $\log F / M_{\mathrm{pul}}-\log P_{\mathrm{pul}}$ of binary $\delta$ Sct stars in systems with $P_{\text {orb }}<13$ days.

the literature we assigned errors following the same method as in the previous correlation (eq. 8). We found 0.003 as an average $F / M_{\text {pul }}$ error value for SB2+E, 0.008 for SB2, 0.059 for $\mathrm{E}, 0.040$ for $\mathrm{SB} 2+\mathrm{EV}$, and 0.007 for $\mathrm{SB} 1+\mathrm{E}$ systems. The sample for the correlation between $F / M_{\text {pul }}-P_{\text {pul }}$ consists of 68 pulsators. In particular, 49 of them belong to semi detached, 14 to detached, and 5 to unclassified systems. In Fig. 12 the fit on the $\log P_{\mathrm{pul}}-\log \frac{F}{M_{\mathrm{pul}}}$ values is shown. The relation between gravitational force per surface gram matter is the following:

$$
\log P_{\mathrm{pul}}=-0.71(2)-0.213(7) \log \frac{F}{M_{\mathrm{pul}}}, \text { with } r=0.49
$$

\section{DISCUSSION AND CONCLUSIONS}

The current study presents the most complete up-to-date catalogue of $\delta$ Sct stars in binaries. $203 \delta$ Sct stars in 199 binary systems are included and they are distinguished according to the available information and their characteristics (i.e. $P_{\text {orb }}$ and Roche geometry configuration). Moreover, demographics for these systems relevant to our knowledge about them, their distribution within the stellar evolutionary diagrams as well as updated correlations between their fundamental properties are also presented.

The sample of this study is divided into two groups according to the orbital period values of the systems, with the limit to be set on $P_{\text {orb }}=13$ days. This limit should be considered as a non-strict one and its assumption was based on the distribution of the systems within the $\log P_{\text {pul }}-\log P_{\text {orb }}$ plot (Fig. 4). For systems with $P_{\text {orb }}$ values greater than 13 days it seems that the $P_{\mathrm{pul}}$ is not affected by the binarity at all, while those having $P_{\text {orb }}<13$ days form a 'band' with a clear linear correlation between orbital and pulsation periods. However, there is no doubt that this arbitrary limit might move to a larger $P_{\text {orb }}$ value, if more systems with $P_{\text {orb }} \sim 13$ days will be discovered. Thus, for the time being, in absence of more such systems, this limit seems to differentiate in a satisfactory way the binary $\delta$ Sct stars, whose dominant oscillation frequency is influenced by the presence of a companion.

The $P_{\text {pul }}-P_{\text {orb }}$ dependence in systems with $P_{\text {orb }}<$ 13 days as well as the limit of $P_{\text {orb }}=13$ days cannot be explained so far with high certainty and with a quantitative way. Speaking qualitatively, we can say that in short-period (i.e. $P_{\text {orb }}<13$ days) detached systems the tidal effects may play an important role to the pulsation frequency modulation. Although tidal potential excites g-mode pulsations (Zahn 2008, 2013; Hambleton et al. 2016), there is probably an influence to the p-modes and/or to the radial mode of the pulsating member of the system. In oEA stars this dependence seems to be more complicated because the mass transfer rate may be also a determining parameter additionally to the tidal interaction influence in the pulsations likely in detached systems.

Updated correlations between $P_{\mathrm{pul}}-P_{\mathrm{orb}}$ according to the geometrical status of the systems with $P_{\text {orb }}<13$ days are also derived. The comparison of equations 1,3 , and 5 with the respective ones of Liakos et al. (2012, eq. 1-3) shows that the correlations have not changed significantly, although the present sample is $\sim 50 \%$ larger than that of 2012. On the other hand, if we compare the decimal forms of $P_{\text {pul }}-P_{\text {orb }}$ correlations (eq. 2, 4, and 6) with the respective one of Soydugan et al. (2006b, eq. 1), we can see that the slope has been changed to almost half of its initial value. The present correlation has to be considered as more accurate since the sample is $\sim 5$ times larger than that of Soydugan et al. (2006b). Finally, since these empirical correlations show that the coefficient of $\log P_{\text {orb }}$ in equations 1 , 3 , and 5 is far away from 1 , regardless of the Roche geometry configuration of the systems, the theoretical approximation made by Zhang et al. (2013, eq. 7) may need further examination.

Figs 5, 6, and 7 show the distributions of the binary $\delta$ Sct stars within the well known $H-R, M-R$, and $T_{\text {eff }}-\log g$ diagrams. From these diagrams, nine pulsators have been found to be slightly evolved lying beyond the TAMS line. On the other hand, four $\delta$ Sct stars which are the primary components of RZ Cas, AS Eri, AO Ser, and VV UMa lie below (but very close to) the ZAMS lines of $M-R$ and $T_{\text {eff }}-\log g$ diagrams, but above the ZAMS in the $H-R$ diagram. Therefore, it can be plausibly concluded that, since more than $\sim 85 \%$ of these stars lie between ZAMS-TAMS limits, the $\delta$ Sct stars in close binaries can be considered as Main-Sequence pulsators. Moreover, in Figs. 6 and 7 it is shown that $\sim 15 \%$ of these stars lie beyond the blue edge of the instability strip, while only $\sim 6 \%$ of them are located below the red edge. Small discrepancies regarding the location of the stars close to the boundaries of the Instability Strip probably come from the different theoretical evolutionary models used for the ISB-ISR lines of the $H-R$ and $T_{\text {eff }}-\log g$ diagrams.

From the present sample it is found that the frequencies range of the currently known $\delta$ Sct stars in binaries is

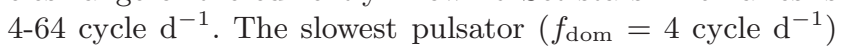
belongs to $\gamma$ Boo, while the fastest one $\left(f_{\text {dom }}=64.4 \mathrm{cy}-\right.$ cle $\mathrm{d}^{-1}$ ) to KIC 11175495 . Unfortunately, the absolute parameters of these two systems have not been calculated to date. However, based on those located outside the Main Sequence limits and mentioned in the previous paragraph, it is found that the less evolved stars pulsate with $f_{\text {dom }}$ values greater than 48 cycle $^{-1}$, except for that of AO Ser. On the other hand, the more evolved pulsators oscillate with $f_{\text {dom }}$ values less than 20 cycle $\mathrm{d}^{-1}$.

The updated correlation between $\log g-\log P_{\text {pul }}$ (eq. 8) 
shows significant change from that derived by Liakos et al. (2012, eq. 5). In the present study, the sample used for this correlation is $\sim 35 \%$ larger than that of 2012 , and, in addition, a new approach regarding their reliability (i.e. errors) was used. For these two reasons, the present correlation can be considered as more realistic and better approximated. Binary $\delta$ Sct stars with $\log g>3.96$ and $\log P_{\text {pul }}<-1.36$ $\left(=f_{\text {dom }}>23\right.$ cycle $\left.\mathrm{d}^{-1}\right)$, i.e. the younger and faster pulsators, follow very well the $P_{\mathrm{pul}}-\log g$ trend (Fig. 11), while the scatter around the fitted line is larger for the older and slower pulsators, especially for those belonging to semi-detached systems. This is probably related to the mass transfer occurrence between the binary members as well as to their evolution. The pulsations in binary $\delta$ Sct stars can potentially begin during the very early stages of their MS life or even before it. According to the distribution shown in Fig. 8, it can be concluded that as the pulsator evolves its dominant pulsation frequency is decreased following the well known frequency evolution of the single pulsating stars. However, at the same time its companion is also evolving, although with slower rate (it should be noted that the pulsators almost always have greater mass than their companions-see Table 1), thus the mass transfer rate is accelerated and the pulsations are certainly affected. Speaking qualitatively, it can be said that probably there is a critical mass gain value of the pulsator after which the correlation between its evolutionary status and its dominant pulsation frequency is agitated. All the above stand for the $\delta$ Sct stars in semi-detached systems of our sample. On the other hand, the pulsators of the detached systems show slightly different behaviour, but it should be noted that the present sample of $\delta$ Sct stars in detached systems is much less than the aforementioned one. However, there are only three cases (CoRoT 105906206, HD 172189, and FL Ori) clearly deviating from the $\log g-\log P_{\text {pul }}$ fit (between $4.4<\log g<3.5$ and $\left.-1.3<\log P_{\text {pul }}<-0.96\right)$, while the three last ones follow it very well. A careful examination of the deviating systems was made, but no obvious similarities (e.g. close mass ratio values) were found. Therefore, with the present sample, the reason of their deviation cannot be explained and more systems have to be added for more certain conclusions.

In order to compare the present correlation between $\log \frac{F}{M_{\mathrm{pul}}}-\log P_{\mathrm{pul}}$ with that derived by Soydugan \& Kaçar (2013, eq. 7) a change in the $P_{\text {pul }}$ measurement units of equation 9 is needed. Therefore, by expressing the $P_{\mathrm{pul}}$ in sec instead of days, the intercept of equation 9 becomes $4.2(6)$. Thus, we can see that the intercepts of the compared equations are quite similar, if we take into account the error bars, but, contrary to that, their slopes differ significantly. We found a slope of $-0.213(7)$, while the respective value of Soydugan \& Kaçar (2013) is $-0.40(6)$. At this point, it has to be noted that the approximations for this correlation are also different between these two studies. We used a sample of 68 stars by taking into account the reliability of their absolute parameters (i.e. errors), while Soydugan \& Kaçar (2013) were based only on the 21 cases with accurate measured absolute parameters. If we use only the stars of our sample with the highest certainty on their absolute parameters (i.e. those that belong to SB2+E systems), then a relation very similar to that of Soydugan \& Kaçar (2013) is derived.
The geometrical configurations of the unclassified eclipsing systems are expected to be determined after a simple light curves analysis. For the ellipsoidal variables and for the spectroscopic binaries, which might also be eclipsing ones but they have not been observed yet, maybe photometric modelling is feasible. Contrary to these, for the spectroscopic non-eclipsing binaries, for the systems whose binarity was discovered by the $O-C$ variation of their $f_{\text {dom }}$, and for the visual binaries we cannot expect any Roche geometry determination.

The most important thing for the future study of this group of stars is undoubtedly the enrichment of the current sample in order to establish further and with higher certainty the present correlations. However, in order to determine more accurately the absolute parameters of the currently known systems, spectroscopic surveys, especially for the eclipsing ones, are strongly recommended, given that the combination of RVs and light curves modellings provides the ultimate knowledge for a system. Theoretical approximations regarding the connection between $P_{\mathrm{pul}}-P_{\text {orb }}$ as well as the limit of 13 days in $P_{\text {orb }}$ are also of high importance, since only a one such study exists so far. Regarding new discoveries, good targets for ground-based photometry and/or spectroscopy can be considered the cases whose pulsations type are unclear (see Table 4). In addition, new observational campaigns and extensive search in the data of space missions such as Kepler are needed to be done in order to discover new cases.

\section{ACKNOWLEDGMENTS}

A.L. acknowledges financial support by the European Space Agency (ESA) under the NELIOTA programme, contract No. 4000112943. This research has made use of NASA's Astrophysics Data System Bibliographic Services and the SIMBAD database, operated at CDS, Strasbourg, France. We thank the anonymous reviewer for the valuable comments that improved the quality of the present work.

\section{REFERENCES}

Abedi A., Riazi N., 2007, Ap\&SS, 307, 409

Abt H. A., 1981, ApJS, 45, 437

Abt H. A., Boonyarak C., 2004, ApJ, 616, 562

Adelman S. J., 1981, A\&AS, 43, 25

Adelman S. J., 1997, A\&AS, 125

Aerts C., Handler G., Arentoft T., Vandenbussche B., Medupe R., Sterken C., 2002, MNRAS, 333, L35

Alecian E., Catala C., van't Veer-Menneret C., Goupil M.-J., Balona L., 2005, A\&A, 442, 993

Alfonso-Garzón J., Domingo A., Mas-Hesse J. M., Giménez A., 2012, A\&A, 548, A79

Alfonso-Garzón J., Montesinos B., Moya A., Mas-Hesse J. M., Martín-Ruiz S., 2014, MNRAS, 443, 3022

Alton K. B., Prša A., 2012, Society for Astronomical Sciences Annual Symposium, 31, 127

Amado P. J., 2007, Communications in Asteroseismology, 151, 57 Amado P. J., Moya A., Suárez J. C., Martín-Ruiz S., Garrido R., Rodríguez E., Catala C., Goupil M. J., 2004, MNRAS, 352, L11

Angione R. J., Sievers J. R., 2006, AJ, 131, 2209

Antoci V., et al., 2014, ApJ, 796, 118 
Antonello E., Mantegazza L., 1986, A\&A, 164, 40

Antonello E., Pasinetti Fracassini L. E., 1998, A\&A, 331, 995

Arentoft T., Sterken C., Handler G., 2001, MNRAS, 326, 192

Arentoft T., Lampens P., van Cauteren P., Duerbeck H. W., García-Melendo E., Sterken C., 2004, A\&A, 418, 249

Arentoft T., Bouzid M. Y., Sterken C., Freyhammer L. M., Frandsen S., 2005, PASP, 117, 601

Auvergne M., Le Contel J.-M., Baglin A., 1979, A\&A, 76, 15

Baade D., Bardelli S., Beaulieu J. P., Vogel S., 1993, A\&A, 269, 195

Balona L. A., et al., 2001, MNRAS, 321, 239

Balona L. A., et al., 2011, MNRAS, 413, 2403

Balona L. A., et al., 2012, MNRAS, 424, 1187

Balona L. A., Daszyńska-Daszkiewicz J., Pamyatnykh A. A., 2015, MNRAS, 452, 3073

Barblan F., Bartholdi P., North P., Burki G., Olson E. C., 1998, A\&AS, 132, 367

Bardin C., Imbert M., 1982, A\&AS, 47, 319

Bartolini C., Piccioni A., Silveri P., 1975, Information Bulletin on Variable Stars, 981

Bartolini C., Dapergolas A., Piccioni A., 1981, Information Bulletin on Variable Stars, 2010

Bartolini C., Grilli F., Parmeggiani G., Piccioni A., Silveri P., 1983, A\&AS, 53, 139

Beardsley W. R., Zizka E. R., 1977, Rev. Mex. Astron. Astrofis., 3,109

Bell S. A., Hilditch R. W., Edwin R. P., 1993, MNRAS, 260, 478

Benisty M., et al., 2011, A\&A, 531, A84

Bertiau F. C., 1957, ApJ, 125, 696

Böhm T., Zima W., Catala C., Alecian E., Pollard K., Wright D., 2009, A\&A, 497, 183

Böhm T., Lignières F., Wade G., Petit P., Aurière M., Zima W., Fumel A., Alecian E., 2012, A\&A, 537, A90

Böhm T., et al., 2015, A\&A, 577, A64

Bossi M., Guerrero G., Mantegazza L., Scardia M., 1983, A\&AS, 53,395

Božić H., Nemravova J., Harmanec P., 2013, Information Bulletin on Variable Stars, 6086

Bradley P. A., Guzik J. A., Miles L. F., Uytterhoeven K., Jackiewicz J., Kinemuchi K., 2015, AJ, 149, 68

Brancewicz H. K., Dworak T. Z., 1980, Acta Astron., 30, 501

Breger M., 2000, in Breger M., Montgomery M., eds, Astronomical Society of the Pacific Conference Series Vol. 210, Delta Scuti and Related Stars. p. 3

Breger M., Hutchins J., Kuhi L. V., 1976, ApJ, 210, 163

Breger M., Pamyatnykh A. A., Zima W., Garrido R., Handler G., Reegen P., 2002, MNRAS, 336, 249

Breger M., Davis K. A., Dukes R. J., 2008, Communications in Asteroseismology, 153, 63

Bruntt H., Southworth J., Torres G., Penny A. J., Clausen J. V., Buzasi D. L., 2006, A\&A, 456, 651

Bruntt H., et al., 2010, A\&A, 512, A55

Budding E., Butland R., 2011, MNRAS, 418, 1764

Budding E., Erdem A., Çiçek C., Bulut İ., Soydugan F., Soydugan E., Bakiş V., Demircan O., 2004, A\&A, 417, 263

Buzasi D. L., et al., 2005, ApJ, 619, 1072

Cakırlı Ö., İbanoğlu C., 2016, New Astron., 45, 36

Calışkan Ş., et al., 2014, AJ, 148, 126

Carrier F., Debernardi Y., Udry S., Carquillat J.-M., Ginestet N., 2002, A\&A, 390, 1027

Casas R., Moya A., Suárez J. C., Martín-Ruíz S., Amado P. J., Rodríguez-López C., Garrido R., 2009, ApJ, 697, 522

Casey M. P., et al., 2013, MNRAS, 428, 2596

Castor J. I., 1968, ApJ, 154, 793

Catanzaro G., Frasca A., Molenda-Żakowicz J., Marilli E., 2010, A\&A, 517, A3

Catanzaro G., et al., 2011, MNRAS, 411, 1167

Caton D. B., 2004, Information Bulletin on Variable Stars, 5531
Chang S.-W., Protopapas P., Kim D.-W., Byun Y.-I., 2013, AJ, 145,132

Chapellier E., et al., 2004, A\&A, 426, 247

Chaubey U. S., 1983, Information Bulletin on Variable Stars, 2348 Chaubey U. S., 1992, Ap\&SS, 189, 263

Christiansen J. L., Derekas A., Ashley M. C. B., Webb J. K., Hidas M. G., Hamacher D. W., Kiss L. L., 2007, MNRAS, 382,239

Conidis G. J., Gazeas K. D., Capobianco C. C., Ogloza W., 2010, Communications in Asteroseismology, 161, 23

Costa J. E. S., et al., 2007, A\&A, 468, 637

Creevey O. L., et al., 2009, A\&A, 507, 901

Creevey O. L., et al., 2010, Astronomische Nachrichten, 331, 952

Da Silva R., Maceroni C., Gandolfi D., Lehmann H., Hatzes A. P., 2014, A\&A, 565, A55

Dal H. A., Sipahi E., 2013, Publ. Astron. Soc. Australia, 30, e016

Dall T. H., Frandsen S., 2002, A\&A, 386, 964

Dallaporta S., Munari U., 2005, ArXiv Astrophysics e-prints,

Dallaporta S., Tomov T., Zwitter T., Munari U., 2002, Information Bulletin on Variable Stars, 5312

Davis P. J., Siess L., Deschamps R., 2013, A\&A, 556, A4

De Cat P., et al., 2007, A\&A, 463, 243

De Mey K., Daems K., Sterken C., 1998, A\&A, 336, 527

Deb S., Tiwari S. K., Singh H. P., Seshadri T. R., Chaubey U. S., 2009, Bulletin of the Astronomical Society of India, 37

Derekas A., et al., 2009, MNRAS, 394, 995

Desikachary K., 1974, A\&A, 35, 303

Dickens R. J., 1967, ApJ, 148, L33

Diethelm R., 1993, Information Bulletin on Variable Stars, 3894

Dimitrov D., Kraicheva Z., Popov V., 2008a, Information Bulletin on Variable Stars, 5842

Dimitrov D., Kraicheva Z., Popov V., 2008b, Information Bulletin on Variable Stars, 5856

Dimitrov D., Kraicheva Z., Popov V., 2009a, Information Bulletin on Variable Stars, 5883

Dimitrov D., Kraicheva Z., Popov V., 2009b, Information Bulletin on Variable Stars, 5892

Dimitrov D., Kraicheva Z., Popov V., Genkov V., 2010, Information Bulletin on Variable Stars, 5925

Doğruel M. B., Gürol B., 2015, New Astron., 40, 20

Dubath P., et al., 2011, MNRAS, 414, 2602

Ducati J. R., Penteado E. M., Turcati R., 2011, A\&A, 525, A26

Dvorak S., 2009, Communications in Asteroseismology, 160, 64

Dworak T. Z., 1973, Acta Astron., 23, 37

Dworak T. Z., Zieba S., 1975, Information Bulletin on Variable Stars, 1005

ESA 1997, VizieR Online Data Catalog, 1239

Eaton J. A., Henry G. W., Odell A. P., 2008, ApJ, 679, 1490

Eggen O. J., 1970, PASP, 82, 274

Eggen O. J., 1979, Information Bulletin on Variable Stars, 1544

Eggleton P. P., Tokovinin A. A., 2008, MNRAS, 389, 869

Eker Z., Bilir S., Soydugan F., Gökçe E. Y., Soydugan E., Tüysüz M., Şenyüz T., Demircan O., 2014, Publ. Astron. Soc. Australia, 31, e024

Ekmekçi F., Topal S., 2008, New Astron., 13, 118

Escolà-Sirisi E., Juan-Samsó J., Vidal-Sáinz J., Lampens P., García-Melendo E., Gómez-Forrellad J. M., Wils P., 2005, A\&A, 434, 1063

Fabricius C., Makarov V. V., 2000, A\&A, 356, 141

Fabricius C., Høg E., Makarov V. V., Mason B. D., Wycoff G. L., Urban S. E., 2002, A\&A, 384, 180

Fedotov Y. T., Gladyr A. V., 1991, Astronomicheskij Tsirkulyar, 1548,21

Fekel F. C., 2015, AJ, 149, 83

Fekel F. C., Tomkin J., Williamson M. H., 2013, AJ, 146, 129

Fox Machado L., Michel R., Álvarez M., Fu J.-N., Zurita C., 2011, in Revista Mexicana de Astronomia y Astrofisica Conference Series. pp 237-238 (arXiv:1103.3071) 
Frandsen S., Jones A., Kjeldsen H., Viskum M., Hjorth J., Andersen N. H., Thomsen B., 1995, A\&A, 301, 123

Frasca A., Catalano S., Mantovani D., 1997, A\&A, 320, 101

Frémat Y., Lampens P., Hensberge H., 2005, MNRAS, 356, 545

Freyhammer L. M., Arentoft T., Sterken C., 2001, A\&A, 368, 580

Fu J.-N., 2000, in Szabados L., Kurtz D., eds, Astronomical Society of the Pacific Conference Series Vol. 203, IAU Colloq. 176: The Impact of Large-Scale Surveys on Pulsating Star Research. pp 475-476

Fu J.-N., Jiang S.-Y., 1999, Delta Scuti Star Newsletter, 13, 9

Fu J.-N., Sterken C., 2003, A\&A, 405, 685

Fu J.-N., et al., 2008, AJ, 135, 1958

Galeev A. I., Ivanova D. V., Shimansky V. V., Bikmaev I. F., 2012, Astronomy Reports, 56, 850

García J., 2012, Journal of the American Association of Variable Star Observers (JAAVSO), 40, 272

Garrido R., Rolland A., López de Coca P., González S. F., Hobart M. A., Peña J. H., 1985, A\&A, 144, 211

Gaulme P., Guzik J. A., 2014, in Guzik J. A., Chaplin W. J., Handler G., Pigulski A., eds, IAU Symposium Vol. 301, Precision Asteroseismology. pp 413-414, doi:10.1017/S1743921313014816

Gazeas K. D., Niarchos P. G., Boutsia K. A., 2004, Communications in Asteroseismology, 144, 26

Gies D. R., Williams S. J., Matson R. A., Guo Z., Thomas S. M., Orosz J. A., Peters G. J., 2012, AJ, 143, 137

Girardi L., Bressan A., Bertelli G., Chiosi C., 2000, A\&AS, 141,371

Golovin A., Pavlenko E., 2007, in Hartkopf W. I., Harmanec P., Guinan E. F., eds, IAU Symposium Vol. 240, Binary Stars as Critical Tools Tests in Contemporary Astrophysics. p. 330 (arXiv:0712.1983)

González J. F., Hubrig S., Savanov I., 2007, Information Bulletin on Variable Stars, 5794

González J. F., Hubrig S., Kurtz D. W., Elkin V., Savanov I., 2008, MNRAS, 384, 1140

Grassitelli L., Fossati L., Langer N., Miglio A., Istrate A. G., Sanyal D., 2015, A\&A, 584, L2

Gregg T. A., Prša A., Welsh W. F., Orosz J. A., Fetherolf T., 2013, in American Astronomical Society Meeting Abstracts \#221. p. 142.12

Griffin R. F., 2012, Journal of Astrophysics and Astronomy, 33,29

Griffin R. F., Boffin H. M. J., 2003, The Observatory, 123, 203

Groenewegen M. A. T., Decin L., Salaris M., De Cat P., 2007, A\&A, 463, 579

Guiglion G., et al., 2013, A\&A, 550, L10

Gunsriwiwat K., Mkrtichian D. E., 2015, Information Bulletin on Variable Stars, 6148

Guo Z., Gies D. R., Matson R. A., García Hernández A., 2016, ApJ, 826, 69

Hambleton K. M., et al., 2013, MNRAS, 434, 925

Hambleton K., et al., 2016, MNRAS,

Hambsch F.-J., Lampens P., van Cauteren P., Kleidis S., Robertson C. W., Krajci T., Wils P., 2010, Information Bulletin on Variable Stars, 5949

Handler G., 2002, in Sterken C., Kurtz D. W., eds, Astronomical Society of the Pacific Conference Series Vol. 256, Observational Aspects of Pulsating B- and A Stars. p. 71

Handler G., Shobbrook R. R., 2002, MNRAS, 333, 251

Hanna M., 2014, Journal of Korean Astronomical Society, 47, 99

Hareter M., et al., 2008, A\&A, 492, 185

Hareter M., et al., 2014, A\&A, 567, A124

Hartkopf W. I., McAlister H. A., 1984, PASP, 96, 105

Hartkopf W. I., Mason B. D., Finch C. T., Zacharias N., Wycoff G. L., Hsu D., 2013, AJ, 146, 76

Hatzes A. P., Mkrtichian D. E., 2004, MNRAS, 351, 663

Henry G. W., Fekel F. C., 2005, AJ, 129, 2026
Henry G. W., Fekel F. C., Kaye A. B., Kaul A., 2001, AJ, 122,3383

Henry G. W., Fekel F. C., Henry S. M., 2004, AJ, 127, 1720

Hildebrandt G., 1992, Astronomische Nachrichten, 313, 233

Hintz E. G., Bush T. C., Rose M. B., 2005, AJ, 130, 2876

Hong K., Lee J.-W., Kim S.-L., Koo J.-R., Lee C.-U., Yushchenko A. V., Kang Y.-W., 2015, AJ, 150, 131

Horan S., 1979, AJ, 84, 1770

Horch E., Franz O. G., Ninkov Z., 2000, AJ, 120, 2638

Hosseinzadeh B., Pazhouhesh R., Yakut K., 2014, New Astron., 27,95

Houdek G., Balmforth N. J., Christensen-Dalsgaard J., Gough D. O., 1999, A\&A, 351, 582

Hube D. P., Aikman G. C. L., 1991, PASP, 103, 49

Huber D., et al., 2014, ApJS, 211, 2

Hudson K. I., Chiu H.-Y., Maran S. P., Stuart F. E., Vokac P. R., 1971, ApJ, 165, 573

Hurta Z., Pocs M. D., Szeidl B., 2007, Information Bulletin on Variable Stars, 5774

İbanoğlu C., et al., 2008, MNRAS, 384, 331

İbanoğlu C., et al., 2009, MNRAS, 392, 757

Jerzykiewicz M., 1972, PASP, 84, 529

Jørgensen H. E., 1975, A\&A, 44, 459

Jørgensen H. E., Gronbech B., 1978, A\&A, 66, 377

Kaye A. B., 1999, Information Bulletin on Variable Stars, 4697

Kaye A. B., Henry G. W., Rodriguez E., 2000, Information Bulletin on Variable Stars, 4850

Keen M. A., Bedding T. R., Murphy S. J., Schmid V. S., Aerts C., Tkachenko A., Ouazzani R.-M., Kurtz D. W., 2015, MNRAS, 454,1792

Khaliullin K. F., Khaliullina A. I., 2012, MNRAS, 419, 3393

Khokhuntod P., Fu J.-N., Boonyarak C., Marak K., Chen L., Jiang S.-Y., 2007, Chinese J. Astron. Astrophys., 7, 421

Khruslov A. V., 2010, Peremennye Zvezdy Prilozhenie, 10

Kim S.-L., Kwon S.-G., Youn J.-H., Mkrtichian D. E., Lee J.-W., 2002, Information Bulletin on Variable Stars, 5314

Kim S.-L., Lee J.-W., Kwon S.-G., Youn J.-H., Mkrtichian D. E., Kim C., 2003, A\&A, 405, 231

Kim S.-L., Kwon S.-G., Lee D.-J., Lee C.-U., Jin H., Lee J.-W., 2004a, in Kurtz D. W., Pollard K. R., eds, Astronomical Society of the Pacific Conference Series Vol. 310, IAU Colloq. 193: Variable Stars in the Local Group. p. 399

Kim S.-L., et al., 2004b, Information Bulletin on Variable Stars, 5537

Kim S.-L., Kang Y.-B., Koo J.-R., Mkrtichian D. E., Lee J.-W., 2004c, Information Bulletin on Variable Stars, 5538

Kim S.-L., Lee J.-W., Lee C.-U., Kang Y.-B., Koo J.-R., Mkrtichian D. E., 2005a, Information Bulletin on Variable Stars, 5598

Kim S.-L., Lee J.-W., Kang Y.-B., Koo J.-R., Mkrtichian D. E., 2005b, Information Bulletin on Variable Stars, 5628

Kim S.-L., Lee J. W., Koo J.-R., Kang Y. B., Mkrtichian D. E., 2005c, Information Bulletin on Variable Stars, 5629

Kim S.-L., Lee C.-U., Koo J.-R., Kang Y.-B., Lee J.-W., Mkrtichian D. E., 2005d, Information Bulletin on Variable Stars, 5669

Kim S.-L., Lee C.-U., Lee J.-W., 2006, Mem. Soc. Astron. Italiana, 77,184

Kim S.-L., Lee J.-W., Lee C.-U., Youn J.-H., 2010a, PASP, 122,1311

Kim D.-W., et al., 2010b, AJ, 139, 757

Kim C., Yushchenko A. V., Kim S.-L., Jeon Y.-B., Kim C.-H., 2012, PASP, 124, 401

Kipp S. L., Russell J. L., Stein J. W., Breakiron A., 1978, AJ, 83,636

Kirk B., et al., 2016, AJ, 151, 68

Kiss L. L., 1995, Information Bulletin on Variable Stars, 4237

Kiss L. L., 2002, Information Bulletin on Variable Stars, 5355 
Kiss L. L., Alfaro E. J., Bakos G., Csak B., Szatmary K., 1999, Information Bulletin on Variable Stars, 4698

Koen C., Eyer L., 2002, MNRAS, 331, 45

Koen C., van Rooyen R., van Wyk F., Marang F., 1999, MNRAS, 309, 1051

Koo J.-R., Lee J. W., Hong K., Kim S.-L., Lee C.-U., 2016, AJ, 151,77

Korda D., Zasche P., Kučáková H., 2015, New Astron., 40, 64

Kreiner J. M., 2004, Acta Astron., 54, 207

Kreiner J. M., Kim C.-H., Nha I.-S., 2001, An Atlas of O-C Diagrams of Eclipsing Binary Stars

Krisciunas K., Simon T., Crowe R. A., Roberts M., 1995, Information Bulletin on Variable Stars, 4264

Kundra E., Hric L., Gális R., 2013, Baltic Astronomy, 22, 111

Kurtz D. W., 1978, ApJ, 221, 869

Kurtz D. W., 1979, MNRAS, 186, 567

Kurtz D. W., 1980, MNRAS, 190, 479

Kurtz D. W., 1984, MNRAS, 206, 253

Kurtz D. W., Elkin V. G., Mathys G., 2007, MNRAS, 380, 741

Kurtz D. W., Hubrig S., González J. F., van Wyk F., Martinez P., 2008, MNRAS, 386, 1750

Kurtz D. W., Hambleton K. M., Shibahashi H., Murphy S. J., Prša A., 2015, MNRAS, 446, 1223

Kusakin A. V., Goranskii V. P., 1995, Astronomy Letters, 21, 180

Lampens P., 1992, Delta Scuti Star Newsletter, 5, 9

Lampens P., Rufener F., 1990, A\&AS, 83, 145

Lampens P., van Camp M., Sinachopoulos D., 1999, Delta Scuti Star Newsletter, 13, 10

Lampens P., van Camp M., Sinachopoulos D., 2000, A\&A, 356,895

Lampens P., van Cauteren P., Strigachev A., Kim S.-L., Kang Y. B., Koo J.-R., Mkrtichian D. E., 2004, Information Bulletin on Variable Stars, 5572

Lampens P., et al., 2005, A\&A, 438, 201

Lampens P., et al., 2011, A\&A, 534, A111

Lampens P., et al., 2013, A\&A, 549, A104

Laney C. D., Joner M., Rodriguez E., 2003, in Sterken C., ed., Astronomical Society of the Pacific Conference Series Vol. 292, Interplay of Periodic, Cyclic and Stochastic Variability in Selected Areas of the H-R Diagram. p. 203

Latković O., 2012, Serbian Astronomical Journal, 184, 77

Lázaro C., Arévalo M. J., Claret A., Rodríguez E., Olivares I., 2001, MNRAS, 325, 617

Lázaro C., Martínez-Pais I. G., Arévalo M. J., 2004, MNRAS, 351,707

Le Contel J. M., Valtier J. C., Sareyan J. P., Baglin A., Zribi G., 1974, A\&AS, 15, 115

Lee J.-W., Kim C.-H., Koch R. H., 2007, MNRAS, 379, 1665

Lee C.-U., Kim S.-L., Lee J.-W., Kim C.-H., Jeon Y.-B., Kim H.-I., Yoon J.-N., Humphrey A., 2008, MNRAS, 389, 1630

Lee C.-U., Kim S.-L., Lee J. W., Pavlovski K., Southworth J., 2012, in Richards M. T., Hubeny I., eds, IAU Symposium Vol. 282, From Interacting Binaries to Exoplanets: Essential Modeling Tools. pp 327-328, doi:10.1017/S1743921311027748

Lee J.-W., Kim S.-L., Hong K., Koo J.-R., Lee C.-U., Youn J.-H., 2016a, AJ, 151, 25

Lee J. W., Hong K., Kim S.-L., Koo J.-R., 2016b, MNRAS, 460,4220

Lehmann H., Scholz G., Hildebrandt G., Yang S., 1997, A\&A, 327,167

Lehmann H., Egorova I., Scholz G., Hildebrandt G., Andrievsky S. M., 2003, A\&A, 402, 229

Lehmann H., et al., 2011, A\&A, 526, A124

Lehmann H., Southworth J., Tkachenko A., Pavlovski K., 2013, A\&A, 557, A79

Lenz P., Daszyńska-Daszkiewicz J., Pamyatnykh A. A., Breger M., 2008, Communications in Asteroseismology, 153, 40

Li L.-J., Qian S.-B., 2013, PASJ, 65
Li Z.-P., Yan X., 2007, PASP, 119, 508

Li Z.-P., et al., 2004, A\&A, 420, 283

Li L.-J., Qian S.-B., Xiang F.-Y., 2010, PASJ, 62, 987

Liakos A., Cagaš P., 2014, Ap\&SS, 353, 559

Liakos A., Niarchos P., 2009, Communications in Asteroseismology, 160, 2

Liakos A., Niarchos P., 2011, Communications in Asteroseismology, 162, 73

Liakos A., Niarchos P., 2012, New Astron., 17, 634

Liakos A., Niarchos P., 2013a, in 11th Hellenic Astronomical Conference. pp 44-44 (arXiv: 1404.3126)

Liakos A., Niarchos P., 2013b, Ap\&SS, 343, 123

Liakos A., Niarchos P., 2015, in Rucinski S. M., Torres G., Zejda M., eds, Astronomical Society of the Pacific Conference Series Vol. 496, Living Together: Planets, Host Stars and Binaries. p. 195 (arXiv: 1506.07409)

Liakos A., Niarchos P., 2016, preprint, (arXiv:1606.08638)

Liakos A., Ulaş B., Gazeas K., Niarchos P., 2008, Communications in Asteroseismology, 157, 336

Liakos A., Zasche P., Niarchos P., 2010, in Prša A., Zejda M., eds, Astronomical Society of the Pacific Conference Series Vol. 435, Binaries - Key to Comprehension of the Universe. p. 101 (arXiv:0910.2812)

Liakos A., Niarchos P., Soydugan E., Zasche P., 2012, MNRAS, 422,1250

Lipski Ł., Stẹpień K., 2008, MNRAS, 385, 481

Liu Y., et al., 1999, Acta Astrophysica Sinica, 19, 201

Liu N., Zhang X.-B., Ren A.-B., Deng L.-C., Luo Z.-Q., 2012, Research in Astronomy and Astrophysics, 12, 671

López de Coca P., Costa V., Rolland A., Walker E. N., 1979, A\&AS, 36, 61

Lowder W. M., 1989, Journal of the American Association of Variable Star Observers (JAAVSO), 18, 116

Lucy L. B., Sweeney M. A., 1971, AJ, 76, 544

Maceroni C., et al., 2014, A\&A, 563, A59

Malkov O. Y., Tamazian V. S., Docobo J. A., Chulkov D. A., 2012, A\&A, 546, A69

Manimanis V. N., Niarchos P. G., 2001, A\&A, 369, 960

Manimanis V. N., Vamvatira-Nakou C., Niarchos P. G., 2009, Ap\&SS, 323, 115

Mantegazza L., Poretti 2005, Communications in Asteroseismology, 146, 33

Mantegazza L., Zerbi F. M., Sacchi A., 2000, A\&A, 354, 112

Mantegazza L., Rainer M., Antonello E., 2010, A\&A, 512, A42

Mantegazza L., et al., 2012, A\&A, 542, A24

Manzoori D., Salar A., 2016, ApJ, 152, 26

Margrave T. E., Peniche R., Pena J. H., 1982, Information Bulletin on Variable Stars, 2240

Martín-Ruiz S., Amado P. J., Suárez J. C., Moya A., Arellano Ferro A., Ribas I., Poretti E., 2005, A\&A, 440, 711

Martinez P., 2002, The Observatory, 122, 359

Martinez P., et al., 1999, MNRAS, 309, 871

Mason B. D., Wycoff G. L., Hartkopf W. I., Douglass G. G., Worley C. E., 2001, AJ, 122, 3466

Mawet D., Mennesson B., Serabyn E., Stapelfeldt K., Absil O., 2011, ApJ, 738, L12

McInally C. J., Austin R. D., 1977, Information Bulletin on Variable Stars, 1334

McNamara D. H., Feltz Jr. K. A., 1976, PASP, 88, 510

Michel E., Goupil M. J., Lebreton Y., Baglin A., 1993, Ap\&SS, 210,181

Michel E., Belmonte J. A., Alvarez M., Jiang S. Y., 1995, Delta Scuti Star Newsletter, 8, 6

Middleton C. T., 2011, Monthly Notes of the Astronomical Society of South Africa, 70, 189

Milone E. F., Wilson W. J. F., Fry D. J. I., Schiller S. J., 1994, PASP, 106, 1120

Mitton J., Stickland D. J., 1979, MNRAS, 186, 189 
Mkritichian D. E., Fedotov Y. T., Romanov Y. S., 1995, Delta Scuti Star Newsletter, 8, 16

Mkrtichian D. E., Gamarova A. Y., 2000, Information Bulletin on Variable Stars, 4836

Mkrtichian D. E., Yurkov A., 1998, in Dusek J., Zejda M., eds, 29th Conference on Variable Star Research. p. 143

Mkrtichian D. E., Kusakin A. V., Gamarova A. Y., Nazarenko V., 2002, in Aerts C., Bedding T. R., Christensen-Dalsgaard J., eds, Astronomical Society of the Pacific Conference Series Vol. 259, IAU Colloq. 185: Radial and Nonradial Pulsationsn as Probes of Stellar Physics. p. 96

Mkrtichian D. E., et al., 2003, in Sterken C., ed., Astronomical Society of the Pacific Conference Series Vol. 292, Interplay of Periodic, Cyclic and Stochastic Variability in Selected Areas of the H-R Diagram. p. 113

Mkrtichian D. E., et al., 2004, A\&A, 419, 1015

Moon T. T., Keay D. M., 1982, Information Bulletin on Variable Stars, 2145

Moon T., van Antwerpen C., 2009, Journal of the American Association of Variable Star Observers (JAAVSO), 37, 3

Moon T. T., Coates D. W., Halprin L., Thompson K., 1983, Information Bulletin on Variable Stars, 2383

Morguleff N., Rutily B., Terzan A., 1976, A\&A, 52, 129

Moriarty D. J. W., Bohlsen T., Heathcote B., Richards T., Streamer M., 2013, Journal of the American Association of Variable Star Observers (JAAVSO), 41, 182

Murphy S. J., Shibahashi H., 2015, MNRAS, 450, 4475

Murphy S. J., et al., 2013a, MNRAS, 432, 2284

Murphy S. J., et al., 2013b, MNRAS, 436, 2877

Murphy S. J., et al., 2015a, Publ. Astron. Soc. Australia, 32, e036

Murphy S. J., Bedding T. R., Niemczura E., Kurtz D. W., Smalley B., 2015b, MNRAS, 447, 3948

Narusawa S.-Y., 2013, PASJ, 65

Narusawa S.-Y., Yamsasaki A., Fukata M., Takeda M., Nakamura Y., Okazaki A., 2002a, PASJ, 54, 139

Narusawa S., Waki Y., Ioroi M., Takeuti M., 2002b, Information Bulletin on Variable Stars, 5279

Natta A., Grinin V. P., Mannings V., Ungerechts H., 1997, ApJ, 491,885

Nichols J. S., Henden A. A., Huenemoerder D. P., Lauer J. L., Martin E., Morgan D. L., Sundheim B. A., 2010, ApJS, 188,473

Norton A. J., Lohr M. E., Smalley B., Wheatley P. J., West R. G., 2016, A\&A, 587, A54

Ohshima O., et al., 2001, AJ, 122, 418

Paparó M., Sterken C., 2000, A\&A, 362, 245

Paparó M., Rodríguez E., McNamara B. J., Kolláth Z., Rolland A., González-Bedolla S. F., Jiang S.-Y., Li Z.-P., 2000, A\&AS, 142,1

Peña J. H., et al., 2015, Rev. Mex. Astron. Astrofis., 51, 51

Peniche R., Peña J. H., 1981, Information Bulletin on Variable Stars, 1970

Peters G. J., Wilson R. E., 2012, in American Astronomical Society Meeting Abstracts \#220. p. 406.04

Petrova A. V., Orlov V. V., 1999, AJ, 117, 587

Pigulski A., Michalska G., 2007, Acta Astron., 57, 61

Pocs M. D., Szeidl B., 2000, Information Bulletin on Variable Stars, 4832

Pojmanski G., 2002, Acta Astron., 52, 397

Polubek G., 2001, Odessa Astronomical Publications, 14

Ponsen J., 1963, Bull. Astron. Inst. Netherlands, 17, 44

Pop A., Turcu V., Marcu A., 2011, Ap\&SS, 333, 17

Popper D. M., 1988, AJ, 95, 190

Poretti E., Buzasi D., Laher R., Catanzarite J., Conrow T., 2002, A\&A, 382, 157

Pourbaix D., et al., 2004, A\&A, 424, 727

Pribulla T., Rucinski S. M., 2006, AJ, 131, 2986
Pribulla T., Kreiner J. M., Tremko J., 2003, Contributions of the Astronomical Observatory Skalnate Pleso, 33, 38

Pribulla T., et al., 2009, AJ, 137, 3655

Proust D., Ochsenbein F., Pettersen B. R., 1981, A\&AS, 44, 179

Prugniel P., Vauglin I., Koleva M., 2011, A\&A, 531, A165

Pustylnik I., Kalv P., Harvig V., 2007, Astronomical and Astrophysical Transactions, 26, 31

Reimers D., 1976, A\&A, 53, 377

Riboni E., Poretti E., Galli G., 1994, A\&AS, 108

Richards J. W., Starr D. L., Miller A. A., Bloom J. S., Butler N. R., Brink H., Crellin-Quick A., 2012, ApJS, 203, 32

Rodríguez E., Breger M., 2001, A\&A, 366, 178

Rodríguez E., Sedano J. L., López de Coca P., Rolland A., 1988, Information Bulletin on Variable Stars, 3174

Rodríguez E., González-Bedolla S. F., Rolland A., Costa V., López de Coca P., 1997, A\&A, 324, 959

Rodríguez E., González-Bedolla S. F., López-González M. J., 1998a, A\&A, 336, 911

Rodríguez E., Rolland A., López-González M. J., Costa V., 1998b, A\&A, 338, 905

Rodríguez E., López-González M. J., López de Coca P., 2000, A\&AS, 144, 469

Rodríguez E., López-González M. J., Rolland A., Costa V., González-Bedolla S. F., 2001, A\&A, 376, 489

Rodríguez E., García J. M., Gamarova A. Y., Costa V., Daszyńska-Daszkiewicz J., López-González M. J., Mkrtichian D. E., Rolland A., 2004, MNRAS, 353, 310

Rodríguez E., et al., 2010, MNRAS, 408, 2149

Rolland A., López de Coca P., Hobart M. A., Peña J. H., Parrao L., 1987, Rev. Mex. Astron. Astrofis., 14, 419

Rucinski S. M., Duerbeck H. W., 2006, AJ, 132, 1539

Rucinski S. M., Pribulla T., van Kerkwijk M. H., 2007, AJ, 134,2353

Santagati G., Rodono M., Ventura R., Poretti E., 1989, Information Bulletin on Variable Stars, 3373

Savanov I. S., Ryabchikova T. A., Davydova E. S., 1996, Astronomy Letters, 22, 815

Schmid V. S., Aerts C., 2016, preprint, (arXiv:1605.07958)

Schmid V. S., et al., 2014, A\&A, 570, A33

Schmid V. S., et al., 2015, A\&A, 584, A35

Senyüz T., Soydugan E., 2008, Communications in Asteroseismology, 157,365

Senyüz T., Soydugan E., 2014, in Guzik J. A., Chaplin W. J., Handler G., Pigulski A., eds, IAU Symposium Vol. 301, Precision Asteroseismology. pp 483-484, doi:10.1017/S1743921313015160

Shibahashi H., Kurtz D. W., 2012, MNRAS, 422, 738

Shibahashi H., Kurtz D. W., Murphy S. J., 2015, MNRAS, 450, 3999

Skarka M., Liška J., Zejda M., Mikulášek Z., 2016, Commmunications of the Konkoly Observatory Hungary, 105, 141

Slawson R. W., et al., 2011, AJ, 142, 160

Smalley B., et al., 2011, A\&A, 535, A3

Smalley B., et al., 2014, A\&A, 564, A69

Sokolovsky K. V., 2009, Peremennye Zvezdy Prilozhenie, 9

Solonovich A. P., Bryukhanov I. S., Semenyuta A. S., 2003, Odessa Astronomical Publications, 16, 66

Southworth J., et al., 2011, MNRAS, 414, 2413

Soydugan E., Kaçar Y., 2013, AJ, 145, 87

Soydugan E., Soydugan F., 2007, in Demircan O., Selam S. O., Albayrak B., eds, Astronomical Society of the Pacific Conference Series Vol. 370, Solar and Stellar Physics Through Eclipses. p. 344

Soydugan E., Soydugan F., İbanoğlu C., Frasca A., Demircan O., Akan M. C., 2006a, Astronomische Nachrichten, 327, 905

Soydugan E., İbanoğlu C., Soydugan F., Akan M. C., Demircan O., 2006b, MNRAS, 366, 1289 
Soydugan E., Soydugan F., Demircan O., İbanoğlu C., 2006c, MNRAS, 370, 2013

Soydugan E., et al., 2008, Communications in Asteroseismology, 157,379

Soydugan E., Soydugan F., Şenyüz T., Püsküllü C., Tüysüz M., Bakıs V., Bilir S., Demircan O., 2009, Information Bulletin on Variable Stars, 5902

Soydugan E., Soydugan F., Şenyüz T., Tüysüz M., Bakiş V., Bilir S., Çiçek C., Demircan O., 2010, in Prša A., Zejda M., eds, Astronomical Society of the Pacific Conference Series Vol. 435, Binaries - Key to Comprehension of the Universe. p. 331

Soydugan E., Soydugan F., Şenyüz T., Püsküllü Ç., Demircan O., 2011, New Astron., 16, 72

Soydugan F., Soydugan E., Kanvermez Ç., Liakos A., 2013, MNRAS, 432, 3278

Soydugan E., Soydugan F., Aliçavuş F., Erdem A., 2016, New Astron., 46, 40

Sterken C., 1977, Information Bulletin on Variable Stars, 1294

Sterken C., Jerzykiewicz M., 1986, A\&A, 169, 164

Sterken C., Jerzykiewicz M., 1989, Information Bulletin on Variable Stars, 3321

Sterken C., et al., 2012, Journal of Astronomical Data, 18

Sterne T. E., 1938, ApJ, 87, 133

Streamer M., Bohlsen T., Ogmen Y., 2016, Journal of the American Association of Variable Star Observers (JAAVSO), 44, 39

Sudar D., Harmanec P., Lehmann H., Yang S., Božić H., Ruždjak D., 2011, A\&A, 528, A146

Sumter G. C., Beaky M. M., 2007, Information Bulletin on Variable Stars, 5798

Szatmary K., 1990, Journal of the American Association of Variable Star Observers (JAAVSO), 19, 52

Tanriver M., 2015, New Astron., 36, 56

Taranova O. G., Shenavrin V. I., 1997, Astronomy Letters, 23, 698

Templeton M. R., Bradley P. A., Guzik J. A., 2000, ApJ, 528, 979

Tkachenko A., Lehmann H., Mkrtichian D. E., 2009, A\&A, 504, 991

Tkachenko A., Lehmann H., Mkrtichian D., 2010, in Prša A., Zejda M., eds, Astronomical Society of the Pacific Conference Series Vol. 435, Binaries - Key to Comprehension of the Universe. p. 245

Todorova P. N., Khruzina T. S., 1989, Information Bulletin on Variable Stars, 3367

Tokovinin A., Hartung M., Hayward T. L., 2010, AJ, 140, 510

Torres K. B. V., Lampens P., Frémat Y., Hensberge H., Lebreton Y., Škoda P., 2011, A\&A, 525, A50

Tsvetkov T. G., 1993, Ap\&SS, 208, 285

Turcu V., Pop A., Moldovan D., 2008, Information Bulletin on Variable Stars, 5826

Turcu V., Pop A., Marcu A., Moldovan D., 2011, Ap\&SS, 331, 105

Turner G., Holaday J., 2013, Journal of the American Association of Variable Star Observers (JAAVSO), 41, 34

Turner G., Holaday J., 2015, Journal of the American Association of Variable Star Observers (JAAVSO), 43, 40

Turner G., Katichuck R., Holaday J., 2014, Journal of the American Association of Variable Star Observers (JAAVSO), 42,134

Turon C., et al., 1993, Bulletin d'Information du Centre de Donnees Stellaires, 43

Ulaş B., Ulusoy C., Gazeas K., Erkan N., Liakos A., 2014, in Guzik J. A., Chaplin W. J., Handler G., Pigulski A., eds, IAU Symposium Vol. 301, Precision Asteroseismology. pp 507-508, doi:10.1017/S1743921313015287

Uytterhoeven K., et al., 2011, A\&A, 534, A125

Valtier J. C., 1972, A\&A, 16, 38

Vander Linden D., Sterken C., 1986, A\&A, 168, 155

Ventura R., Catanzaro G., Christensen-Dalsgaard J., di Mauro M. P., Paternò L., 2007, MNRAS, 381, 1647
Veto B., Kovacs G., 1981, Information Bulletin on Variable Stars, 2030

Volkov I. M., 1990, Information Bulletin on Variable Stars, 3493

Volkov I., Volkova N., 2010, in Prša A., Zejda M., eds, Astronomical Society of the Pacific Conference Series Vol. 435, Binaries - Key to Comprehension of the Universe. p. 323

Vyas M. L., Abhyankar K. D., 1989, A\&AS, 81, 67

Walczak P., Szewczuk W., Daszyńska-Daszkiewicz J., 2012, Astronomische Nachrichten, 333, 1065

Walker E. N., Jones D. H. P., 1969, The Observatory, 89, 202

Wang S.-M., Qian S.-B., Li L.-J., Zhu L.-Y., Zhao E.-G., Zhou X., 2015, New Astron., 34, 11

Wehlau W., Wehlau A., 1995, in Stobie R. S., Whitelock P. A., eds, Astronomical Society of the Pacific Conference Series Vol. 83, IAU Colloq. 155: Astrophysical Applications of Stellar Pulsation. p. 323

Weiss W. W., et al., 1998, A\&A, 338, 919

Wonnacott D., Kellett B. J., Stickland D. J., 1993, MNRAS, 262,277

Woollands R. M., Pollard K. R., Ramm D. J., Wright D. J., Böhm T., 2013, MNRAS, 432, 327

Yang D., Tang Q., Jiang S., Wang H., 1993, Information Bulletin on Variable Stars, 3831

Yang Y.-G., Hu S.-M., Guo D.-F., Wei J.-Y., Dai H.-F., 2010, AJ, 139,1360

Yang Y.-G., Wei J.-Y., Li H.-L., 2014, AJ, 147, 35

Yang Y., Zhang L., Dai H., Li H., 2015, New Astron., 37, 48

Zahn J.-P., 2008, in Goupil M.-J., Zahn J.-P., eds, EAS Publications Series Vol. 29, EAS Publications Series. pp 67-90 (arXiv: 0807.4870), doi:10.1051/eas:0829002

Zahn J.-P., 2013, in Souchay J., Mathis S., Tokieda T., eds, Lecture Notes in Physics, Berlin Springer Verlag Vol. 861, Lecture Notes in Physics, Berlin Springer Verlag. p. 301, doi:10.1007/978-3-642-32961-6'8

Zakirov M. M., 1998, Astronomy Letters, 24, 808

Zasche P., 2011, New Astron., 16, 157

Zasche P., Uhlař R., 2013, MNRAS, 429, 3472

Zejda M., et al., 2010, preprint, (arXiv:1012.5679)

Zejda M., Paunzen E., Baumann B., Mikulášek Z., Liška J., 2012, A\&A, 548, A97

Zhang X.-B., Zhang R.-X., Li Q.-S., 2009, Research in Astronomy and Astrophysics, 9, 422

Zhang X.-B., Deng L. C., Luo C.-Q., 2012, AJ, 144, 141

Zhang X.-B., Luo C.-Q., Fu J.-N., 2013, ApJ, 777, 77

Zhang X.-B., et al., 2014, AJ, 148, 106

Zhang X.-B., Luo Y.-P., Wang K., 2015a, AJ, 149, 96

Zhang X.-B., Luo Y.-P., Wang K., Luo C.-Q., 2015b, AJ, 150, 37

Zhiping L., 2000a, A\&A, 360, 185

Zhiping L., 2000b, A\&A, 362, 595

Zhou A.-Y., 2001a, Information Bulletin on Variable Stars, 5087

Zhou A.-Y., 2001b, Information Bulletin on Variable Stars, 5096

Zhou A.-Y., 2002, A\&A, 385, 503

Zhou A.-Y., 2010, preprint, (arXiv:1002.2729)

Zhou A.-Y., Jiang S.-Y., 2011, AJ, 142, 100

Zhou A.-Y., Rodríguez E., Rolland A., Costa V., 2001, MNRAS, 323, 923

Zhu L.-Y., Qian S.-B., Xiang F.-Y., 2006, PASJ, 58, 361

Zola S., et al., 2016, AJ, 152, 33

Zorec J., Royer F., 2012, A\&A, 537, A120

Zwitter T., Munari U., Marrese P. M., Prša A., Milone E. F., Boschi F., Tomov T., Siviero A., 2003, A\&A, 404, 333

This paper has been typeset from a $\mathrm{T}_{\mathrm{E}} \mathrm{X} / \mathrm{LAT}_{\mathrm{E}} \mathrm{X}$ file prepared by the author.

MNRAS 000, 1-20 (201X) 